MATHEMATICS OF COMPUTATION

Volume 68, Number 228, Pages 1533-1567

S 0025-5718(99)01092-3

Article electronically published on March 10, 1999

\title{
COMPOSITE WAVELET BASES FOR OPERATOR EQUATIONS
}

\author{
WOLFGANG DAHMEN AND REINHOLD SCHNEIDER
}

\begin{abstract}
This paper is concerned with the construction of biorthogonal wavelet bases defined on a union of parametric images of the unit $n$-cube. These bases are to satisfy certain requirements imposed by applications to a class of operator equations acting on such domains. This covers also elliptic boundary value problems, although this study is primarily motivated by our previous analysis of wavelet methods for pseudo-differential equations with special emphasis on boundary integral equations. In this case it is natural to model the boundary surface as a union of parametric images of the unit cube. It will be shown how to construct wavelet bases on the surface which are composed of wavelet bases defined on each surface patch. Here the relevant properties are the validity of norm equivalences in certain ranges of Sobolev scales, as well as appropriate moment conditions.
\end{abstract}

\section{INTRODUCTION}

1.1. Background and motivation. Wavelets that are defined in a classical environment such as the full Euclidean space or the periodic setting exhibit several remarkable features that make them very attractive for the numerical treatment of partial differential as well as integral equations. Due to the paramount importance of Fourier techniques for the construction of wavelets as well as for their analysis one encounters, however, severe obstructions when trying to construct bases with similar favorable properties for more general domain geometries arising in connection with operator equations. The objective of this paper is to provide for domains of practical relevance wavelet bases whose properties will be described below.

The principal features that make wavelet concepts interesting can be roughly summarized as follows.

(a) Norm equivalences. Weighted sequence norms of wavelet expansion coefficients are equivalent to certain function norms. To be more specific, suppose that $\Psi=\left\{\psi_{\lambda}: \lambda \in \nabla\right\}$ is a Riesz basis for $L_{2}$. Here $\nabla$ denotes a suitable index set whose elements $\lambda$ typically have the form $\lambda=(j, k)$, and $|\lambda|:=j$ refers to the scale, which for simplicity will always correspond to a meshsize of order $2^{-j}$. The index $k$ will generally again be comprised of several indices expressing the type of the wavelet and the location of its support.

Received by the editor December 20, 1996 and, in revised form, December 12, 1997.

1991 Mathematics Subject Classification. Primary 65Y20,68Q25, 65F35, 45L10, 65M99, $76 \mathrm{D} 07$.

Key words and phrases. Biorthogonal wavelets, norm equivalences, boundary element methods, composite multiresolution, multiscale methods for partial differential equations.

The work of the first author has been supported in part by DFG grant Da 117/8-2.

The work of the second author has been supported in part by DFG grant SFB 393.

(C)1999 American Mathematical Society 
It is known [10] that the Riesz basis property implies the existence of a dual Riesz basis $\tilde{\Psi}$, i.e., $\left\langle\psi_{\lambda}, \tilde{\psi}_{\lambda^{\prime}}\right\rangle=\delta_{\lambda, \lambda^{\prime}}, \lambda, \lambda^{\prime} \in \nabla$, where $\langle\cdot, \cdot\rangle$ denotes a scalar product in $L_{2}$. Under ideal circumstances the Riesz basis property is actually only a special case of the following norm equivalences for a whole range of spaces-namely, for some $\gamma, \tilde{\gamma}>0$ one has

$$
\|v\|_{H^{s}} \sim\left(\sum_{\lambda \in \nabla} 2^{2 s|\lambda|}\left|\left\langle v, \tilde{\psi}_{\lambda}\right\rangle\right|^{2}\right)^{\frac{1}{2}}, s \in(-\tilde{\gamma}, \gamma),
$$

where $\gamma$ and $\tilde{\gamma}$ limit the Sobolev regularity of the elements in $\Psi$ and $\tilde{\Psi}$, respectively. Here and in the following $a \sim b$ means that $a \lesssim b$ and $b \lesssim a$, where the latter means that $b$ can be bounded by some constant times $a$ uniformly with respect to any parameters on which $a$ and $b$ may depend.

(b) Cancellation properties. The inner products $\left|\left\langle v, \psi_{\lambda}\right\rangle\right|$ are small when the function $v$ is smooth on the support $\Omega_{\lambda}$ of $\psi_{\lambda}$. In quantitative terms one has, for instance, when the wavelets have vanishing polynomial moments of order $\tilde{d}$,

$$
\left|\left\langle v, \psi_{\lambda}\right\rangle\right| \lesssim 2^{-|\lambda|(\tilde{d}+n / 2)}|v|_{W_{\infty}^{\tilde{d}}\left(\Omega_{\lambda}\right)},
$$

where $|\cdot|_{W_{\infty}^{\tilde{d}}\left(\Omega_{\lambda}\right)}$ denotes the usual $L_{\infty}$-Sobolev semi-norm and $n$ is the spatial dimension. We deliberately avoid the vanishing moments as the primary concept, since they may be cumbersome to describe for manifolds. What matters are the approximation properties of the spaces spanned by the dual system $\tilde{\Psi}$.

(c) Fast transforms. For certain purposes the (usually more local) singlescale representation of trial functions is preferable, so that fast well-conditioned transforms from single-scale to multi-scale representations are needed. Stability requires the Riesz basis property, while efficiency of both transforms in the sense that the computational work stays proportional to the size of the transformed arrays follows from the locality of the elements in $\Psi$ and $\tilde{\Psi}$.

To motivate the subsequent development let us add a few comments on how the properties (a), (b) and (c) come into play when trying to use wavelet concepts for solving an operator equation of the form $A u=f$ for any given $f \in H^{-t}$. Here we assume that for a given bounded domain or manifold $\Omega$ which admits the definition of Sobolev spaces $H^{s}$ for a certain range of $s$ (see [1]). $H^{s}$ is to be understood for $s<0$ as the dual $\left(H^{-s}\right)^{*}$ of $H^{-s}$. When $\Omega$ is a bounded domain the definition of $H^{s}$ may incorporate (homogeneous) boundary conditions. Furthermore, we assume that $A$ is a boundedly invertible operator from $H^{t}$ onto $H^{-t}$, i.e.,

$$
\|A v\|_{H^{-t}} \sim\|v\|_{H^{t}}
$$

The following examples fit into this setting.

Differential operators. A typical case arises when $\Omega$ is a bounded domain and $A=-\nabla \cdot \mathbf{B} \nabla$, where $\mathbf{B}$ is a uniformly positive matrix on $\Omega$, or $A=-\nabla \cdot \mathbf{B} \nabla+c \mathbf{I}$, $c>0$, in which case $t=1$ and $H^{1}=H_{0}^{1}(\Omega), H^{1}(\Omega)$, respectively.

Boundary integral equations. More generally, we are interested in solving $A u=f$ for operators of the form

$$
(A u)(x)=\int_{\Gamma} K(x, y) u(y) \mathrm{d} y,
$$


where $\Gamma$ is a boundary manifold of dimension $n$ and the kernel $K(\cdot, \cdot)$ satisfies estimates of the type

$$
\left|\partial_{x}^{\alpha} \partial_{y}^{\beta} K(x, y)\right| \leq c_{\alpha, \beta} \operatorname{dist}_{\Gamma}(x, y)^{-(n+2 t+|\alpha|+|\beta|)} .
$$

In this case we require that (1.1.3) holds for Sobolev spaces defined on $\Gamma$. Examples are the single and double layer potential or the hypersingular operator with $t=-1 / 2,0,1 / 2$, respectively, obtained e.g. when transforming an exterior boundary value problem for Laplace's equation into an integral equation, see e.g. [19]. More generally, kernels of this type arise e.g. in connection with computing electrostatic fields, scattering from 3D obstacles, transmission problems, and high quality computer visualization based on the radiosity concept. Again (1.1.3) holds for the respective values of $t$.

We will consider Galerkin schemes based on the trial spaces $S_{J}:=\operatorname{span}\left\{\psi_{\lambda}\right.$ : $|\lambda| \leq J\}$. The relevance of (a) lies in the fact that under the assumption (1.1.3) the scaled stiffness matrices $\mathbf{A}_{\Lambda}:=\left(2^{-t\left(|\lambda|+\left|\lambda^{\prime}\right|\right)}\left\langle A \psi_{\lambda^{\prime}}, \psi_{\lambda}\right\rangle\right)_{\lambda, \lambda^{\prime} \in \Lambda}$ have for any trial space $\operatorname{span}\left\{\psi_{\lambda}: \lambda \in \Lambda\right\}$ uniformly bounded condition numbers [17, 12, 13, 21], provided that

$$
\gamma, \tilde{\gamma}>|t| .
$$

When $A$ is a differential operator, the stiffness matrix with respect to a wavelet basis is less sparse (in a strict sense) than the stiffness matrix with respect to a typical nodal (single-scale) basis. Since only the application of the preconditioned matrix matters in an iterative process, one can use the fact that when $\mathbf{T}$ takes the wavelet coefficients into the nodal coefficients, the stiffness matrix $\mathbf{A}_{W}$ relative to the wavelet basis is related to the stiffness matrix $\mathbf{A}_{N}$ relative to the nodal basis by $\mathbf{A}_{W}=\mathbf{T}^{T} \mathbf{A}_{N} \mathbf{T}$. Thus, even though only the sparse matrix $\mathbf{A}_{N}$ is assembled, property (c) facilitates the efficient application of the preconditioned matrix.

When $A$ is an operator with global Schwartz kernel of the type (1.1.5), the situation is different. Now the stiffness matrix is densely populated. But whereas essentially all entries in $\mathbf{A}_{N}$ are significant, it was observed in [2] that most entries in $\mathbf{A}_{W}$ are so small that the application of a properly sparsified or compressed matrix causes a controllable error. Moreover, rather than considering a fixed threshold independent of the size $N$ of the matrix $\mathbf{A}_{W}$ as in [2], an asymptotic analysis based on (a) and (b) was carried out in $[15,16,17,23,25]$ that culminates in the following (simplified) statement: $\mathbf{A}_{W}$ can be compressed to the order of $N$ nonzero entries so that the asymptotic accuracy of the perturbed system is still of the same order as that of the solution to the unperturbed system provided that the wavelet bases $\Psi, \tilde{\Psi}$ are suitably chosen. Here suitable means that in addition to (1.1.6) one has

$$
\tilde{d}>d-2 t
$$

when

$$
\inf _{v_{j} \in S_{j}}\left\|v_{j}-v\right\|_{L_{2}(\Gamma)} \lesssim 2^{-j d}\|v\|_{H^{d}(\Gamma)}, \quad v \in H^{d}(\Gamma) .
$$

Thus, the cancellation property should be sufficiently strong relative to the order $d$ of the trial spaces and the order $2 t$ of the operator. Note that, in particular, one needs $\tilde{d}>d$ when $t \leq 0$, which rules out orthogonal wavelets in this case, stressing the importance of the more flexible concept of biorthogonality. 
Note that the last comments primarily refer to efficient solvers for essentially uniform refinements; that is, the trial spaces are spanned by all wavelets up to some scale $|\lambda|=J$. On the other hand, (a) suggests tracking only those coefficients of the solution which are needed to represent it within a desired accuracy, while (b) says that in many cases the number of these coefficients can be expected to be relatively small. A natural approach is to adaptively generate possibly lacunary sets $\Lambda \subset \nabla$ containing the most significant wavelet coefficients. In [9] the convergence in the energy norm of such schemes could be established rigorously without an apriori assumption on the unknown solution (such as the saturation property that is commonly assumed in analogous investigations in a finite element context). Again (a) and (b) serve as the main ingredients of the analysis.

1.2. The objectives. In this paper we construct biorthogonal wavelet bases for domains which can be represented as an essentially disjoint union of smooth parametric images of the unit $n$-cube $\square:=[0,1]^{n}$ so that the above mentioned properties (a) - (c) hold to the following extent:

- All wavelets as well as their duals have local support whose diameter is proportional to the respective level of resolution. Hence (c) holds.

- For any order $d$ of accuracy in (1.1.8) of the primal wavelets the cancellation property (1.1.2) holds for any desired $\tilde{d} \geq d$, so that the compression requirements (1.1.7) are met.

- The norm equivalences (1.1.1) hold for $\gamma=3 / 2, \tilde{\gamma}=1 / 2$ even when dealing with nonplanar Lipschitz manifolds. Thus zero order and first order operators (such as the double layer potential and hypersingular operator) as well as second order operators are covered in the above sense.

- The primal and dual wavelets are globally continuous. Hence in certain cases the norm equivalences (1.1.1) extend beyond $-1 / 2$ into the negative range, so that then also the interesting case of the single layer potential operator is covered.

- Norm equivalences with respect to a somewhat different scale of spaces hold for the range $-3 / 2<s<3 / 2$.

The envisaged class of domains is relevant for different types of problems. In the context of boundary integral equations it agrees with already existing elaborate surface modeling techniques employed in Computer Aided Geometric Design [20, 24]. It fits into domain decomposition concepts for boundary value problems on Euclidean domains as well [4]. In general the quality of the bases depends also on the parametric mappings describing the domain partition. Strong unisotropies and distortions are of course expected to cause adverse effects.

The difficulty with extending (1.1.1) further into the negative range (thereby missing an 'optimal' treatment of the single layer potentials) is caused by the fact that biorthogonality is always realized with respect to a modified $L_{2}$-inner product, so that duality may give rise to a scale of dual spaces $H_{s}$ that differ from $\left(H^{-s}\right)^{*}$ for $s \leq-1 / 2$. However, since $-1 / 2$ is just the border case for the range of norm equivalences, the adverse effect on preconditioning for the single layer potential operator is expected to be mild.

1.3. Organization of material. The starting point is that wavelet bases on the interval are by now well understood, see e.g. $[8,14]$. Thus it is natural to form tensor products to deal with cubes, so that the main task is now to form globally 
continuous wavelets on the union of parametric images of such cubes. The glueing technique pursued in this paper relies on a number of specific properties of the univariate ingredients. Therefore Section 2 is devoted to preparing these foundations. We can heavily rely on corresponding recent results from [14]. Nevertheless, there are two additional issues that are essential and therefore have to be taken care of, namely:

- realizing suitable boundary conditions in order to deal with patch interfaces;

- realizing certain symmetry properties of the bases on the interval in order to achieve invariance under similarity transformations of the cube and thereby keep the construction as independent as possible of local reparametrizations and the global topology of the manifold or domain.

Although these issues by themselves are not the central objective of this paper, this material is necessary for the understanding and concrete realization of the subsequent construction. However, in order not to interrupt the flow of development too severely we have exported some of the proofs to an appendix.

In Section 3 we carry the univariate results over to the unit cube essentially in a routine way. This serves mainly to set some necessary notation and to establish certain estimates that will be needed later for the stability analysis pertaining to (1.1.1).

Section 4 is concerned with the construction of globally continuous wavelets on the type of manifolds that will be described at the beginning of this section. The construction proceeds in the following steps. First we construct globally continuous biorthogonal generator bases on the manifold. By the boundary and symmetry properties of the univariate ingredients this amounts to glueing only those generators on adjacent patches which refer to the same knot on the common interface. As for the subsequent construction of corresponding wavelets, the basic point of view is that each complement basis spanning a complement between two successive multiresolution spaces corresponds to a matrix completion of the refinement matrices for the generator bases $[5,26]$. Again due to the properties of the univariate bases we then exploit the fact that certain such completions can be explicitly constructed from the univariate ingredients. With the aid of the techniques from [5] we are then able to project these initial completions into those corresponding to the desired wavelet bases. We obtain explicit representations of the wavelet filters in a way that makes it conceptually very easy to incorporate in addition homogeneous Dirichlet boundary conditions into the multiresolution spaces. Finally, employing results from [10] we establish the norm equivalences and cancellation properties announced above.

During the reviewing process we became aware of the manuscript by C. Canuto, A. Tabacco, K. Urban, The wavelet element method, Part I, Istituto di Analisi Numerica, Pavia, Preprint, 1997, to appear in Appl. Comput. Harmonic Anal., where similar bases are constructed in a completely different way.

\section{Biorthogonal MUltiresolution on the interval}

A core ingredient of the whole construction is suitable biorthogonal wavelet bases on the unit interval. This section is devoted to deriving the relevant properties. The main issues are boundary conditions and symmetry properties, both of which will be essential for the construction of composite bases. 
2.1. Multiresolution sequences. To set the stage, we begin by recalling some results from [14] which are necessary for the understanding of the subsequent developments. The common approach to biorthogonal multiresolution on $[0,1]$ begins with some dual pair $(\theta, \tilde{\theta})$ of refinable functions, i.e.,

$$
\theta(x)=\sum_{k \in \mathbb{Z}} a_{k} \theta(2 x-k), \quad \tilde{\theta}(x)=\sum_{k \in \mathbb{Z}} \tilde{a}_{k} \tilde{\theta}(2 x-k),
$$

and

$$
\langle\theta, \tilde{\theta}(\cdot-k)\rangle_{\mathbb{R}}=\int_{\mathbb{R}} \theta(x) \tilde{\theta}(x-k) \mathrm{d} x=\delta_{0, k}, \quad k \in \mathbb{Z} .
$$

The idea is then to construct (the primal) spaces $S_{j}$ on $[0,1]$ by taking those translates $\theta\left(2^{j} \cdot-k\right)$ which are supported inside $[0,1]$ supplemented by certain additional linear combinations of the translates overlapping the end points of the interval. These linear combinations are formed in such a way that the resulting span still contains all polynomials of a desired order (see $[8,14]$ ). To our knowledge, only in [14] do the dual multiresolution spaces $\tilde{S}_{j}$ induced by $\tilde{\theta}$ also exhibit the original order of polynomial exactness which is crucial in the present context. It is well known that the order of polynomial exactness determines the approximation order of the spaces.

An important family of initial dual pairs is based on B-splines. Denoting by $\left[x_{0}, \ldots, x_{d}\right] f$ the $d$ th order divided difference of $f$ at the points $x_{0}, \ldots, x_{d} \in \mathbb{R}$ (see e.g. [3]), the $d$ th order centered cardinal B-spline is defined by $\theta(x)={ }_{d} \theta(x):=$ $d[0,1, \ldots, d]\left(\cdot-x-\left\lfloor\frac{d}{2}\right\rfloor\right)_{+}^{d-1}$, where $x_{+}^{l}:=(\max \{0, x\})^{l}$ and $\lfloor x\rfloor(\lceil x\rceil)$ is the largest (smallest) integer less (greater) than or equal to $x$. Thus $\theta$ is centered around $\frac{\mu(d)}{2}$, where $\mu(d):=d \bmod 2$, and has support $\operatorname{supp}_{d} \theta=\left[-\left\lfloor\frac{d}{2}\right\rfloor,\left\lceil\frac{d}{2}\right\rceil\right]:=$ $\left[\ell_{1}, \ell_{2}\right]$. The B-spline ${ }_{d} \theta$ is well-known to be refinable with finitely supported real mask.

It has been shown in [7] that for each $d$ and any $\tilde{d} \geq d, \tilde{d} \in \mathbb{N}$, such that $d+\tilde{d}$ is even, there exists a function ${ }_{d, \tilde{d}} \tilde{\theta}$ which is also refinable and has support

$$
\operatorname{supp}_{d, \tilde{d}} \tilde{\theta}=\left[\ell_{1}-\tilde{d}+1, \ell_{2}+\tilde{d}-1\right]=:\left[\tilde{\ell}_{1}, \tilde{\ell}_{2}\right]
$$

so that ${ }_{d} \theta$ and ${ }_{d, \tilde{d}} \tilde{\theta}$ form a dual pair, i.e.,

$$
\left\langle{ }_{d} \theta,{ }_{d, \tilde{d}} \tilde{\theta}(\cdot-k)\right\rangle_{\mathbb{R}}=\delta_{0, k}, \quad k \in \mathbb{Z}
$$

Moreover, we shall exploit the fact that they both share the same symmetry properties

$$
{ }_{d} \theta(x+\mu(d))={ }_{d} \theta(-x), \quad{ }_{d, \tilde{d}} \tilde{\theta}(x+\mu(d))={ }_{d, \tilde{d}} \tilde{\theta}(-x), \quad x \in \mathbb{R} .
$$

Finally, ${ }_{d, \tilde{d}} \tilde{\theta}$ is exact of order $\tilde{d}$, i.e., all polynomials of degree less than $\tilde{d}$ can be represented as linear combinations of the translates ${ }_{d, \tilde{d}} \tilde{\theta}(\cdot-k), k \in \mathbb{Z}$. It is also known that the regularity of ${ }_{d, \tilde{d}} \tilde{\theta}$ increases proportionally with $\tilde{d}$.

In the following $d, \tilde{d}$ will be arbitrary as above but fixed, so we can suppress them as indices and write briefly $\theta, \tilde{\theta}$ if there is no risk of confusion.

We next briefly recall from [14] pairs of generator bases $\Theta_{j}, \tilde{\Theta}_{j}$ which span multiresolution sequences of spaces $S_{j}([0,1]), \tilde{S}_{j}([0,1])$ which are exact of order $d, \tilde{d}$, respectively. These collections have the form

$$
\Theta_{j}^{\prime}=\Theta_{j}^{L} \cup \Theta_{j}^{I} \cup \Theta_{j}^{R}, \quad \tilde{\Theta}_{j}^{\prime}=\tilde{\Theta}_{j}^{L} \cup \tilde{\Theta}_{j}^{I} \cup \tilde{\Theta}_{j}^{R} .
$$


Setting $g_{[j, k]}:=2^{j / 2} g\left(2^{j} \cdot-k\right)$, the sets $\Theta_{j}^{I}$, $\tilde{\Theta}_{j}^{I}$ consist of the interior basis functions $\theta_{[j, k]}, \tilde{\theta}_{[j, k]}, k \in \Delta_{j}^{I}, \tilde{\Delta}_{j}^{I}$, respectively, which do not interfere with the end points of the interval. Here the corresponding index sets are

$$
\begin{array}{r}
\tilde{\Delta}_{j}^{I}:=\left\{\tilde{\ell}, \ldots, 2^{j}-\tilde{\ell}-\mu(d)\right\}, \quad \Delta_{j}^{I}=\left\{\ell, \ldots, 2^{j}-\ell-\mu(d)\right\}, \\
\ell:=\tilde{\ell}-(\tilde{d}-d) .
\end{array}
$$

To ensure that the interior functions are indeed fully supported in $[0,1], \tilde{\ell}$ has only to be bounded from below by

$$
\tilde{\ell} \geq \tilde{\ell}_{2}
$$

see (2.1.1). In addition one needs the collections

$$
\Theta_{j}^{X}=\left\{\theta_{j, k}^{X}: k \in \Delta_{j}^{X}\right\}, \quad \tilde{\Theta}_{j}^{X}=\left\{\tilde{\theta}_{j, k}^{X}: k \in \tilde{\Delta}_{j}^{X}\right\}
$$

of boundary adapted basis functions, where for $X \in\{L, R\}$

$$
\tilde{\Delta}_{j}^{L}:=\{\tilde{\ell}-\tilde{d}, \ldots, \tilde{\ell}-1\}, \quad \tilde{\Delta}_{j}^{R}:=\left\{2^{j}-\tilde{\ell}+1-\mu(d), \ldots, 2^{j}-\tilde{\ell}+\tilde{d}-\mu(d)\right\},
$$

and

$$
\Delta_{j}^{L}=\{\ell-d, \ldots, \ell-1\}, \quad \Delta_{j}^{R}=\left\{2^{j}-\ell+1-\mu(d), \ldots, 2^{j}-\ell+d-\mu(d)\right\} .
$$

The functions $\theta_{j, k}^{L}, \tilde{\theta}_{j, k}^{L}$ are certain fixed linear combinations of the translates $\left.\theta_{[j, k]}\right|_{[0,1]},\left.\tilde{\theta}_{[j, k]}\right|_{[0,1]}$ chosen so as to ensure that the linear spans of the collections $\Theta_{j}^{\prime}$ and $\tilde{\Theta}_{j}^{\prime}$ contain all polynomials of degree less than $d, \tilde{d}$, respectively. Specifically, we set

$$
\theta_{j, \ell-d+r}^{L}:=\left.\sum_{m=-\ell_{2}+1}^{\ell-1} \tilde{\alpha}_{m, r} \theta_{[j, m]}\right|_{[0,1]}, \quad \tilde{\theta}_{j, \tilde{\ell}-\tilde{d}+l}^{L}:=\left.\sum_{m=-\tilde{\ell}_{2}+1}^{\tilde{\ell}-1} \alpha_{m, l} \tilde{\theta}_{[j, m]}\right|_{[0,1]},
$$

for $r=0, \ldots, d-1$ and $l=0, \ldots, \tilde{d}-1$, respectively, where $\alpha_{m, l}:=\left\langle(\cdot)^{l}, \theta(\cdot-m)\right\rangle_{\mathbb{R}}$, $\tilde{\alpha}_{m, r}:=\left\langle(\cdot)^{r}, \tilde{\theta}(\cdot-m)\right\rangle_{\mathbb{R}}$ (see [14] for details on the evaluation of these quantities).

On account of the symmetry properties of $\theta$ and $\tilde{\theta}$ (2.1.3), one can show that suitable generator basis functions adapted to the right end of the interval can be defined via symmetry by

$$
\begin{array}{ll}
\theta_{j, 2^{j}-\ell+d-\mu(d)-r}^{R}(1-x):=\theta_{j, \ell-d+r}^{L}(x), & r=0, \ldots, d-1, \\
\tilde{\theta}_{j, 2^{j}-\tilde{\ell}+\tilde{d}-\mu(d)-r}^{R}(1-x):=\tilde{\theta}_{j, \tilde{\ell}-\tilde{d}+r}^{L}(x), & r=0, \ldots, \tilde{d}-1,
\end{array}
$$

while we will also frequently exploit the fact that for $m \in \Delta_{j}^{I}$ (respectively $\tilde{\Delta}_{j}^{I}$ )

$$
\phi_{[j, m]}(x)=\phi_{\left[j, 2^{j}-m-\mu(d)\right]}(1-x), \quad \phi=\theta, \tilde{\theta} .
$$

To make sure that the collections of boundary functions $\Theta_{j}^{X}, \tilde{\Theta}_{j}^{X}$ are separated we will assume in the following that $j \geq\left\lceil\log _{2}\left(\tilde{\ell}+\tilde{\ell}_{2}-1\right)+1\right\rceil=: j_{0}$.

Defining for any set $\Theta$ of functions in $L_{2}(\Omega)$

$$
S(\Theta):=\cos _{L_{2}}(\operatorname{span} \Theta),
$$

the following facts have been proved in [14].

Proposition 2.1.1. Let $\Theta_{j}^{\prime}, \tilde{\Theta}_{j}^{\prime}$ be given by (2.1.4). 
1. The spaces $S_{j,[0,1]}:=S\left(\Theta_{j}^{\prime}\right)$ and $\tilde{S}_{j,[0,1]}:=S\left(\tilde{\Theta}_{j}^{\prime}\right)$ are nested, i.e., $S_{j,[0,1]} \subset$ $S_{j+1,[0,1]}, \tilde{S}_{j,[0,1]} \subset \tilde{S}_{j+1,[0,1]}, j \geq j_{0}$.

2. The spaces $S_{j,[0,1]}, \tilde{S}_{j,[0,1]}$ are exact of order $d, \tilde{d}$, respectively, i.e.,

$$
\Pi_{d}([0,1]) \subset S_{j,[0,1]}, \quad \Pi_{\tilde{d}}([0,1]) \subset \tilde{S}_{j,[0,1]}, \quad j \geq j_{0},
$$

where $\Pi_{d}$ denotes the space of polynomials of order $d$.

The nestedness of the spaces $S\left(\Theta_{j}^{\prime}\right)$ and $S\left(\tilde{\Theta}_{j}^{\prime}\right)$ follows from the fact that also the boundary functions satisfy two-scale refinement relations whose exact format is given in [14]. Of course, the refinement filters of the boundary functions differ from those of the interior functions. However, there are only finitely many of them, namely $d, \tilde{d}$ for each end of the interval, respectively, and the filter coefficients are independent of the level $j$.

Nevertheless, in the absence of translation invariance, it will be extremely convenient to view the collections $\Theta_{j}^{\prime}$ or $\tilde{\Theta}_{j}^{\prime}$ as (column) vectors whose entries are the respective basis functions. More generally, we will extend this convention in a canonical way to any other collections of functions $\Theta$ or $\Phi$ in some Hilbert space $\mathcal{H}$ with inner product $\langle\cdot, \cdot\rangle$ which will arise below. Specifically,

$$
\langle\Theta, \Phi\rangle:=(\langle\theta, \phi\rangle)_{\theta \in \Theta, \phi \in \Phi}
$$

will denote a matrix. Thus $\langle\Theta, \Phi\rangle$ is a column or row vector when $\Phi$ or $\Theta$, respectively, consists of only one element. Likewise the fact that, due to refinability, each $\theta_{j, k}$ can be written as a linear combination of elements in $\Theta_{j+1}^{\prime}$ means that these refinement relations can be conveniently expressed by a matrix relation between $\Theta_{j}^{\prime}$ and $\Theta_{j+1}^{\prime}$ (and likewise for the collections $\tilde{\Theta}_{j}^{\prime}$ ) of the following form:

$$
\Theta_{j}^{\prime T}=\left(\Theta_{j+1}^{\prime}\right)^{T} \mathbf{M}_{j, 0}^{\prime}, \quad\left(\tilde{\Theta}_{j}^{\prime}\right)^{T}=\left(\tilde{\Theta}_{j+1}^{\prime}\right)^{T} \tilde{\mathbf{M}}_{j, 0}^{\prime} .
$$

Thus the $i$ th column of $\mathbf{M}_{j, 0}^{\prime}, \tilde{\mathbf{M}}_{j, 0}^{\prime}$ consists of the filter or mask coefficients of the $i$ th element of $\Theta_{j}^{\prime}, \tilde{\Theta}_{j}^{\prime}$, respectively. As mentioned above, the dependence of the refinement matrices $\mathbf{M}_{j, 0}^{\prime}, \tilde{\mathbf{M}}_{j, 0}^{\prime}$ on $j$ is very weak in the sense that there are only finitely many different coefficients, whose numbering but not whose values depend on $j$. In fact, the refinement matrices have a stationary interior block which grows with $j$, and an upper left and lower right block of fixed size which corresponds to the boundary functions. Moreover, these blocks are symmetric in that the lower right block is obtained from the upper left one by reversing the order of rows and columns - this is an immediate consequence of (2.1.9). Again see [14] for details.

2.2. Boundary conditions for generator bases on $[0,1]$. Due to the boundary modifications, the collections $\Theta_{j}^{\prime}$ and $\tilde{\Theta}_{j}^{\prime}$ are no longer biorthogonal. However, it has been shown in [14] that these collections can always be biorthogonalized, a fact we shall make essential use of. In [14] this has been realized by a change of basis in $\tilde{\Theta}_{j}^{\prime}$. Here we will depart somewhat from this strategy in order to deal with additional requirements concerning boundary conditions. To describe this, note first that by construction the interior functions in $\Theta_{j}^{I}$ and $\tilde{\Theta}_{j}^{I}$ are still biorthogonal. Therefore they will be left essentially untouched, and it suffices to confine the change of bases to the collections of boundary functions. Since generally $\tilde{d} \geq d$, we will always tacitly assume in the sequel that the primal collections $\Theta_{j}^{X}, X \in\{L, R\}$, are extended by the corresponding number $\tilde{d}-d$ of interior functions to match the 
size of $\tilde{\Theta}_{j}^{X}$. To formulate the main observation we denote for any matrix $\mathbf{M}$ the matrix which is obtained by reversing the order of rows and columns of $\mathbf{M}$ by $\mathbf{M} \downarrow$ and recall from $[14,18]$ the following facts.

Proposition 2.2.1. The matrices

$$
\mathbf{T}_{X}:=\left\langle\Theta_{j}^{X}, \tilde{\Theta}_{j}^{X}\right\rangle_{[0,1]}
$$

are independent of $j$. Moreover, one has

$$
\operatorname{det} \mathbf{T}_{X} \neq 0, \quad \operatorname{det} \mathbf{T}_{X}^{0} \neq 0,
$$

where $\mathbf{T}_{X}^{0}$ is the submatrix of $\mathbf{T}_{X}$ obtained by discarding the first row and column.

These facts will allow us to control the behavior of the generator basis functions at the end points of the interval. This is essential for a later construction of composite bases.

Proposition 2.2.2. There exist $\tilde{d} \times \tilde{d}$ matrices $\mathbf{C}_{X}, \tilde{\mathbf{C}}_{X}$, for $X \in\{L, R\}$, independent of $j \geq j_{0}$, satisfying

$$
\mathbf{C}_{R}=\mathbf{C}_{L}^{\uparrow}, \quad \tilde{\mathbf{C}}_{R}=\tilde{\mathbf{C}}_{L}^{\uparrow},
$$

such that the collections

$$
\Theta_{X, j}:=\mathbf{C}_{X} \Theta_{j}^{X}, \quad \tilde{\Theta}_{X, j}:=\tilde{\mathbf{C}}_{X} \tilde{\Theta}_{j}^{X},
$$

satisfy

$$
\left\langle\Theta_{X, j}, \tilde{\Theta}_{X, j}\right\rangle=\mathbf{I}, \quad X \in\{L, R\} .
$$

Denoting the sets of new boundary functions by

$$
\Theta_{X, j}=\left\{\theta_{j, k}: k \in \Delta_{j}^{X}\right\}, \quad \tilde{\Theta}_{X, j}=\left\{\tilde{\theta}_{j, k}: k \in \Delta_{j}^{X}\right\},
$$

and likewise

$$
\theta_{j, k}:=\theta_{[j, k]}, \quad k \in \Delta_{j}^{I}, \quad \tilde{\theta}_{j, k}:=\tilde{\theta}_{[j, k]}, \quad k \in \tilde{\Delta}_{j}^{I},
$$

one has for

$$
\Theta_{j}:=\Theta_{L, j} \cup \Theta_{j}^{I} \cup \Theta_{R, j}, \quad \tilde{\Theta}_{j}:=\tilde{\Theta}_{L, j} \cup \tilde{\Theta}_{j}^{I} \cup \tilde{\Theta}_{R, j},
$$

that

$$
\left\langle\Theta_{j}, \tilde{\Theta}_{j}\right\rangle_{[0,1]}=\mathbf{I},
$$

and the collections $\Theta_{j}, \tilde{\Theta}_{j}$ are uniformly stable in the sense that

$$
\|\mathbf{c}\|_{\ell_{2}\left(\Delta_{j}\right)} \sim\left\|\mathbf{c}^{T} \Theta_{j}\right\|_{L_{2}([0,1])}
$$

(and analogously for $\tilde{\Theta}_{j}$ ) holds uniformly in $j$ and the coefficient sequences $\mathbf{c}$.

Moreover, the following boundary conditions hold:

$$
\begin{gathered}
\theta_{j, k}(0)=\tilde{\theta}_{j, k}(0)=\theta_{j, k}(1)=\tilde{\theta}_{j, k}(1)=0, \\
k \in \Delta_{j} \backslash\left\{\ell-d, 2^{j}-\ell(d)-\ell+d\right\},
\end{gathered}
$$

while

$$
\theta_{j, \ell-d}(0)=\theta_{j, 2^{j}-\ell(d)-\ell+d}(1)=2^{j / 2}, \quad \tilde{\theta}_{j, \tilde{\ell}-\tilde{d}}(0)=\tilde{\theta}_{j, 2^{j}-\ell(d)-\tilde{\ell}+\tilde{d}}(1)=2^{j / 2} \tau,
$$

where

$$
\tau=\operatorname{det} \mathbf{T}_{L}^{0} / \operatorname{det} \mathbf{T}_{L}
$$



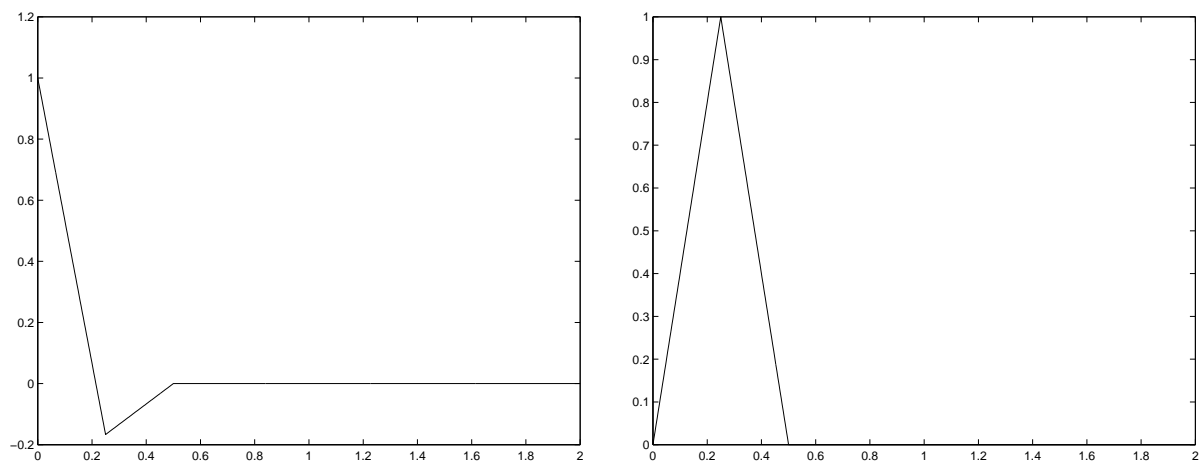

Figure 1. Primal generators $\theta_{j, k}^{L}, k=0,1$, for $d=\tilde{d}=\ell=\tilde{\ell}=2$ at the left boundary
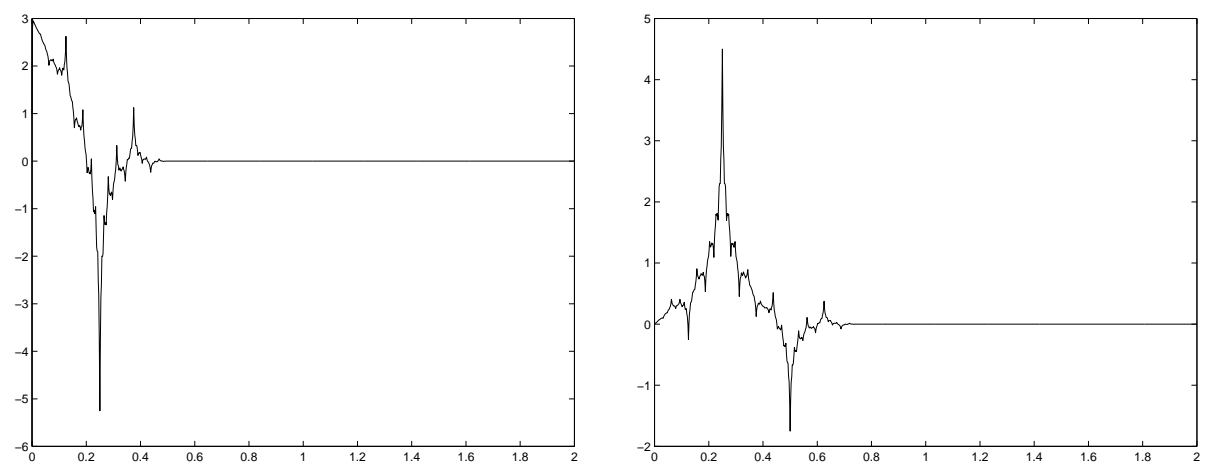

Figure 2. Dual generators $\tilde{\theta}_{j, k}^{L}, k=0,1, d=\tilde{d}=\ell=\tilde{\ell}=2$, at the left boundary

The proof of Proposition 2.2.2 is deferred to the Appendix.

Examples of the boundary adapted generator basis functions for $d=\tilde{d}=\ell=\tilde{\ell}=$ 2 (see (2.1.5), (2.1.6) for the definition of these parameters) are displayed in Figures 1 and 2. The properties (2.2.8) and (2.2.9) readily facilitate adjoining generator basis functions across patch interfaces, since at most one function from each patch has to be glued. This is illustrated below in Figure 3 (at the end of $\S 4.2$ ) for the case of $d=\tilde{d}=2$ and two adjacent intervals.

\subsection{Symmetry.}

Remark 2.3.1. It immediately follows from (2.2.2) and (2.1.9) that the biorthogonalized boundary functions in $\Theta_{X, j}, \tilde{\Theta}_{X, j}$ inherit the symmetry properties from (2.1.9).

It is now easy to derive from the refinement relations of the collections $\Theta_{j}^{\prime}, \tilde{\Theta}_{j}^{\prime}$ the new refinement matrices $\mathbf{M}_{j, 0}, \tilde{\mathbf{M}}_{j, 0}$ for the biorthogonalized bases $\Theta_{j}, \tilde{\Theta}_{j}$. For instance, one has $\mathbf{M}_{j, 0}=\mathbf{C}_{j+1}^{-T} \mathbf{M}_{j, 0}^{\prime} \mathbf{C}_{j}^{T}$, where the matrices $\mathbf{C}_{j}$ are obtained by padding a suitably sized identity matrix with the blocks $\mathbf{C}_{L}$ and $\mathbf{C}_{R}$ from Proposition 2.2.2; see [14] for further details. From Remark 2.3.1, (2.2.2), (2.1.9) and (2.1.10) one concludes the following facts. 
Remark 2.3.2. The refinement matrices $\mathbf{M}_{j, 0}, \tilde{\mathbf{M}}_{j, 0}$ in

$$
\Theta_{j}^{T}=\Theta_{j+1}^{T} \mathbf{M}_{j, 0}, \quad \tilde{\Theta}_{j}^{T}=\tilde{\Theta}_{j+1}^{T} \tilde{\mathbf{M}}_{j, 0}
$$

also satisfy

$$
\mathbf{M}_{j, 0}^{\uparrow}=\mathbf{M}_{j, 0}, \quad \tilde{\mathbf{M}}_{j, 0}^{\uparrow}=\tilde{\mathbf{M}}_{j, 0} .
$$

Moreover, one infers from (2.2.8), (2.2.9) that

$$
\left(\tilde{\mathbf{M}}_{j, 0}\right)_{k, k^{\prime}}=\left(\mathbf{M}_{j, 0}\right)_{k, k^{\prime}}=0
$$

for

$$
\begin{cases}k=\ell-d, & k^{\prime} \neq k, \\ k=2^{j+1}-\mu(d)-\ell+d, & k^{\prime} \neq 2^{j}-\mu(d)-\ell+d,\end{cases}
$$

that is, the refinement relation of basis functions vanishing at the boundary involve only basis functions on the next finer scale which also vanish at the boundary.

As for the symmetry of corresponding wavelets, we recall first from [14] that for any $d+\tilde{d}$ even, $\tilde{d} \geq d$, and corresponding biorthogonal generator bases $\Theta_{j}, \tilde{\Theta}_{j}$ as above one can construct wavelet bases

$$
\Psi_{j}=\left\{\psi_{j, k}: k=1, \ldots 2^{j}\right\}, \quad \tilde{\Psi}_{j}=\left\{\tilde{\psi}_{j, k}: k=1, \ldots, 2^{j}\right\}
$$

which are biorthogonal:

$$
\left\langle\Psi_{j}, \tilde{\Psi}_{j^{\prime}}\right\rangle_{[0,1]}=\delta_{j, j^{\prime}} \mathbf{I}, \quad j, j^{\prime} \geq j_{0} .
$$

Moreover, expressing the corresponding two-scale relations in matrix form as

$$
\Psi_{j}^{T}=\Theta_{j+1}^{T} \mathbf{M}_{j, 1}, \quad \tilde{\Psi}_{j}^{T}=\tilde{\Theta}_{j+1}^{T} \tilde{\mathbf{M}}_{j, 1}
$$

one has

$$
\tilde{\mathbf{M}}_{j, e}^{T} \mathbf{M}_{j, e^{\prime}}=\delta_{e, e^{\prime}} \mathbf{I}, \quad e, e^{\prime} \in\{0,1\} .
$$

Each column of any of the matrices $\mathbf{M}_{j, e}, \tilde{\mathbf{M}}_{j, e}$ contains only a uniformly bounded number of nonzero coefficients forming the filters of the wavelets and generator basis functions whose supports therefore satisfy

$$
\operatorname{diam} \operatorname{supp} \psi_{j, k}, \operatorname{diam\operatorname {supp}} \tilde{\psi}_{j, k}, \operatorname{diam} \operatorname{supp} \theta_{j, k}, \operatorname{diam\operatorname {supp}} \tilde{\theta}_{j, k} \sim 2^{-j} .
$$

However, the particular matrices $\mathbf{M}_{j, 1}, \tilde{\mathbf{M}}_{j, 1}$ constructed in [14] do not necessarily share the same symmetry properties as the refinement matrices in Remark 2.3.2. One can check that the construction in [14] does give

$$
\mathbf{M}_{j, 1}^{\uparrow}=\mathbf{M}_{j, 1}, \quad \tilde{\mathbf{M}}_{j, 1}^{\uparrow}=\tilde{\mathbf{M}}_{j, 1},
$$

when $d$ is even. Let us point out first how to arrange (2.3.9) also when $d$ is odd.

Proposition 2.3.1. Given $\mathbf{M}_{j, 0}, \tilde{\mathbf{M}}_{j, 0}$ from (2.3.1), let $\mathbf{M}_{j, 1}^{\prime}, \tilde{\mathbf{M}}_{j, 1}^{\prime}$ be the particular matrices constructed in [14] satisfying (2.3.7) as above. Then there always exist matrices $\mathbf{M}_{j, 1}, \tilde{\mathbf{M}}_{j, 1}$ which also satisfy (2.3.7) and inherit the same sparseness properties of $\mathbf{M}_{j, 1}^{\prime}, \tilde{\mathbf{M}}_{j, 1}^{\prime}$, so that

$$
\left\|\left(\mathbf{M}_{j, 0}, \mathbf{M}_{j, 1}\right)\right\|,\left\|\left(\tilde{\mathbf{M}}_{j, 0}, \tilde{\mathbf{M}}_{j, 1}\right)\right\|=\mathcal{O}(1)
$$

while in addition

$$
\mathbf{M}_{j, 1}^{\uparrow}=\mathbf{M}_{j, 1}, \quad \tilde{\mathbf{M}}_{j, 1}^{\uparrow}=\tilde{\mathbf{M}}_{j, 1} .
$$


A proof of Proposition 2.3.1 can be found in the Appendix.

In the sequel we will always refer to the symmetric version when dealing with (2.3.7) and (2.3.6).

2.4. Boundary conditions for wavelets on $[0,1]$. Recall that our ultimate goal is to generate wavelets on $\Gamma$ by glueing functions on adjacent patches together in order to realize global continuity. While, as pointed out above, according to (2.2.8) and (2.2.9), this poses no problem for generator basis functions, a simple dimension argument reveals that glueing wavelets that do not vanish on a common patch boundary will not result in a basis for the full complement spaces. However, things would be very simple if there existed wavelet bases whose elements all vanish at the end points of the interval. Unfortunately, according to the following remark which we state here without proof, this is not possible for the above construction of biorthogonal complements.

Remark 2.4.1. There exist no biorthogonal wavelet bases $\Psi_{j}, \tilde{\Psi}_{j}$ for the spaces $S\left(\Theta_{j}\right), S\left(\tilde{\Theta}_{j}\right)$ as above such that all elements of $\Psi_{j}$ vanish at 0 and 1.

One could try to construct directly additional wavelets across interfaces to come up with full complement bases. However, since this would depend strongly on the local mesh topology, we will pursue here a different approach, based on the concepts in [5], that will automatically generate the right wavelets across patch boundaries for any given mesh topology. See [22] for an alternative approach in the case $d=\tilde{d}=2$, where successive projections are used.

A crucial ingredient will be some auxiliary bases that span a complement of $S\left(\Theta_{j}\right)$ in $S\left(\Theta_{j+1}\right)$ such that all their elements do vanish at the end points of the interval. We will point out next how to generate these bases. In view of the envisaged boundary conditions and the structure of the index sets (2.1.7), (2.1.8), we will employ the following univariate functionals:

$$
\tilde{\lambda}_{j, \ell-d}(f):=2^{-j / 2} f(0), \quad \tilde{\lambda}_{j, 2^{j}-\mu(d)-\ell+d}(f):=2^{-j / 2} f(1),
$$

and set $\tilde{\Theta}_{j, 0}:=\tilde{\Theta}_{j} \backslash\left\{\tilde{\theta}_{j, \tilde{\ell}-\tilde{d}}, \tilde{\theta}_{j, 2^{j}-\mu(d)-\tilde{\ell}+\tilde{d}}\right\}$.

Lemma 2.4.1. The collections of functionals

$$
\tilde{\Lambda}_{j}:=\left\{\tilde{\lambda}_{j, \ell-d}, \tilde{\lambda}_{j, 2^{j}-\mu(d)-\ell+d}\right\} \cup \tilde{\Theta}_{j, 0}
$$

$$
\left\langle\Theta_{j}, \tilde{\Lambda}_{j}\right\rangle_{[0,1]}=\mathbf{I}
$$

so that

$$
P_{j} f:=\left\langle f, \tilde{\Lambda}_{j}\right\rangle_{[0,1]} \Theta_{j}
$$

are projectors onto $S\left(\Theta_{j}\right)$. Moreover, one has

$$
\left\|\left\langle\Phi_{j+1}, \tilde{\Lambda}_{j}\right\rangle_{[0,1]}\right\|=\mathcal{O}(1), \quad j \rightarrow \infty,
$$

where $\|\mathbf{A}\|$ denotes the spectral norm of the matrix $\mathbf{A}$.

Proof. The biorthogonality of $\Theta_{j}$ and $\tilde{\Lambda}_{j}$ is an immediate consequence of (2.2.6) and the boundary conditions (2.2.8), (2.2.9) combined with the definition (2.4.2). (2.4.5) follows from the fact that the number and modulus of nonvanishing entries in each row and column of $\left\langle\Theta_{j+1}, \tilde{\Lambda}_{j}\right\rangle_{[0,1]}$ are uniformly bounded in $j$. 
We wish to find bases $\breve{\Psi}_{j}$ for the spaces

$$
W\left(\Lambda_{j}\right):=\left(P_{j+1}-P_{j}\right) S\left(\Phi_{j+1}\right) .
$$

Combining Lemma 2.4.1 with Theorem 3.3 in [5] yields the following result.

Remark 2.4.2. Let $\mathbf{M}_{j, 1}, \tilde{\mathbf{M}}_{j, 1}$ be the matrices constructed in Proposition 2.3.1, and set

$$
\breve{\mathbf{M}}_{j, 1}:=\left(\mathbf{I}-\mathbf{M}_{j, 0}\left\langle\Theta_{j+1}, \tilde{\Lambda}_{j}\right\rangle_{[0,1]}^{T}\right) \mathbf{M}_{j, 1}, \quad \breve{\mathbf{M}}_{j}:=\left(\mathbf{M}_{j, 0}, \breve{\mathbf{M}}_{j, 1}\right) .
$$

Then the inverse $\breve{\mathbf{G}}_{j}=\left(\begin{array}{c}\breve{G}_{j, 0} \\ \breve{\mathbf{G}}_{j, 1}\end{array}\right)=\breve{\mathbf{M}}_{j}^{-1}$ is given by

$$
\breve{\mathbf{G}}_{j, 0}=\tilde{\mathbf{M}}_{j, 0}^{T}+\left\langle\Theta_{j+1}, \tilde{\Lambda}_{j}\right\rangle_{[0,1]}^{T} \mathbf{M}_{j, 1} \tilde{\mathbf{M}}_{j, 1}^{T}, \quad \breve{\mathbf{G}}_{j, 1}=\tilde{\mathbf{M}}_{j, 1}^{T} .
$$

Moreover, by construction, one has

$$
\breve{\mathbf{M}}_{j}^{\uparrow}=\breve{\mathbf{M}}_{j}, \quad \breve{\mathbf{G}}_{j}^{\uparrow}=\breve{\mathbf{G}}_{j} .
$$

The collections

$$
\breve{\Psi}_{j}^{T}=\Theta_{j+1}^{T} \breve{\mathbf{M}}_{j, 1}
$$

span the spaces $W\left(\Lambda_{j}\right)$, i.e., $W\left(\Lambda_{j}\right)=S\left(\breve{\Psi}_{j}\right)$.

For later use we record the following important consequences of the above facts.

Remark 2.4.3. The only nonzero entry in the first and last row of $\mathbf{M}_{j, 0}$ and $\tilde{\mathbf{M}}_{j, 0}$ occurs at the first, respectively last position. The first and last rows of $\breve{\mathbf{M}}_{j, 1}$ have only zero entries.

Proof. The first part of the assertion has already been established in (2.3.3). Combining this with the fact that, by construction, all elements in the complement spaces $W\left(\Lambda_{j}\right)$ defined in (2.4.6) vanish at zero and one, we can prove the rest of the assertion.

\section{WAVELETS ON THE UNIT CUBE}

3.1. Tensor products. The next step is to take tensor products of the univariate constructions. This follows mostly canonical lines, and one only has to fix some notation. We will apply the following rules: Super- or subscripts $\square$ indicate quantities defined on the unit $n$-cube $\square:=(0,1)^{n}$, usually obtained as tensor products. For instance, $\Delta_{j}^{\square}=\Delta_{j} \times \cdots \times \Delta_{j}$. Likewise, $\mathbf{k}$ is to be understood as a multiindex $\mathbf{k}=\left(k_{1}, \ldots, k_{n}\right)$ whenever it is associated with a multivariate quantity. The wavelets now require a further index $\mathbf{e} \in\{0,1\}^{n}$, namely

$$
\psi_{j, \mathbf{e}, \mathbf{k}}^{\square}(\mathbf{x})=\psi_{j, e_{1}, k_{1}}\left(x_{1}\right) \cdots \psi_{j, e_{n}, k_{n}}\left(x_{n}\right),
$$

where

$$
\psi_{j, e, k}= \begin{cases}\theta_{j, k}, k \in \Delta_{j}, & \text { if } \quad e=0, \\ \psi_{j, k}, k \in \nabla_{j}, & \text { if } \quad e=1,\end{cases}
$$

and it will be convenient to identify the indices of the complement basis functions with the set

$$
\nabla_{j}:=\left\{1 / 2, \ldots, 2^{j}-1 / 2\right\} .
$$

It will therefore sometimes be convenient to write $\psi_{j, \mathbb{Q}, \mathbf{k}}^{\square}$ instead of $\theta_{j, \mathbf{k}}^{\square}$, i.e., not to use an extra notation for the scaling functions. 
The matrices $\mathbf{M}_{j}^{\square}, \mathbf{G}_{j}^{\square}$ are now naturally blocked into components $\mathbf{M}_{j, \mathbf{e}}^{\square}, \mathbf{G}_{j, \mathbf{e}}^{\square}$, $\mathbf{e} \in\{0,1\}^{n}$, where

$$
\left(\mathbf{M}_{j, \mathbf{e}}^{\square}\right)_{\mathbf{k}, \mathbf{k}^{\prime}}=\left(\mathbf{M}_{j, e_{1}}\right)_{k_{1}, k_{1}^{\prime}} \cdots\left(\mathbf{M}_{j, e_{n}}\right)_{k_{n}, k_{n}^{\prime}},
$$

and $\mathbf{M}_{j, 1}^{\square}$ is comprised of all the components $\mathbf{M}_{j, \mathbf{e}}^{\square}, \mathbf{e} \in\{0,1\}^{n} \backslash\{\mathbb{D}\}$, while $\mathbf{M}_{j, \mathbb{Q}}^{\square}$ is the refinement matrix of $\Theta_{j}^{\square}$.

3.2. Direct and inverse estimates. Tensor products of functionals defined on univariate functions are canonically defined by their action on the respective variables. Defining in this sense the collections $\tilde{\Lambda}_{j}^{\square}$ based on (2.4.2), we will make crucial use of the projectors

$$
P_{j}^{\square} f:=\left\langle f, \tilde{\Lambda}_{j}^{\square}\right\rangle_{\square} \Theta_{j}^{\square}
$$

onto the spaces $S\left(\Theta_{j}^{\square}\right)$. To describe their relevant properties, let us denote by

$$
\partial \Delta_{j}^{\square}:=\left\{k \in \Delta_{j}^{\square}: k_{i} \in\left\{\ell-d, 2^{j}-\mu(d)-\ell+d\right\}, \text { for some } 1 \leq i \leq n\right\}
$$

and analogously by $\partial \tilde{\Delta}_{j}$ the set of indices associated with the boundary of $\square$. The following facts will be needed later for the stability analysis of the wavelet bases.

Lemma 3.2.1. The projectors $P_{j}^{\square}$ have the following properties.

(i) Whenever $\mathbf{k} \in \Delta_{j}^{\square}$ belongs to $\partial \Delta_{j}^{\square}$, then the quantities $\left\langle f, \tilde{\lambda}_{j, \mathbf{k}}^{\square}\right\rangle_{\square}$ depend only on values of $f$ restricted to the intersection of all faces of $\square$ which $\mathbf{k}$ is associated with. In particular, one has that $P_{j}^{\square}$ interpolates at the vertices of $\square$, i.e., $\left(P_{j}^{\square} f\right)(\mathbf{e})=f(\mathbf{e}), \mathbf{e} \in\{0,1\}^{n}$.

(ii) When $d>n / 2$ the projectors $P_{j}^{\square}$ have the following approximation properties:

$$
\left\|f-P_{j}^{\square} f\right\|_{L_{2}(\square)} \lesssim 2^{-d j}\|f\|_{H^{d}(\square)}, \quad f \in H^{d}(\square) .
$$

Proof. (i) is an immediate consequence of the definition (2.4.2) of the functionals in $\tilde{\Lambda}_{j}$.

The basic idea for proving (ii) is also familiar. Only the fact that functionals in $\tilde{\Lambda}_{j}$ are not all bounded on $L_{2}(\square)$ deserves a little care. To this end, let

$$
\sigma_{j, \mathbf{k}}:=\operatorname{supp} \theta_{j, \mathbf{k}}^{\square}, \quad \Delta_{j, \mathbf{k}}:=\left\{\mathbf{k}^{\prime} \in \Delta_{j}^{\square}: \sigma_{j, \mathbf{k}} \cap \sigma_{j, \mathbf{k}^{\prime}} \neq \emptyset\right\},
$$

and

$$
\hat{\sigma}_{j, \mathbf{k}}:=\bigcup_{\mathbf{k}^{\prime} \in \Delta_{j, \mathbf{k}}} \operatorname{supp} \tilde{\theta}_{j, \mathbf{k}^{\prime}}
$$

Note that

$$
\# \Delta_{j, \mathbf{k}}=\mathcal{O}(1), \quad \operatorname{diam} \hat{\sigma}_{j, \mathbf{k}} \sim 2^{-j}, \quad j \in \mathbb{N} .
$$

By construction, $S\left(\Theta_{j}^{\square}\right)$ contains the space $\Pi_{j, \square}$ of all polynomials of total (even coordinate) degree less than $d$ on $\square$. Since $P_{j}^{\square}$ is a projector, one has $P_{j}^{\square} p=p$ for all $p \in \Pi_{j, \square}$. Hence for any $f \in H^{s}(\square), s>n / 2$, and any $p \in \Pi_{j, \square}$, one has

$$
\begin{aligned}
\left\|f-P_{j}^{\square} f\right\|_{L_{2}\left(\sigma_{j, \mathbf{k}}\right)}^{2} & \lesssim\|f-p\|_{L_{2}\left(\sigma_{j, \mathbf{k}}\right)}^{2}+\left\|P_{j}^{\square}(f-p)\right\|_{L_{2}\left(\sigma_{j, \mathbf{k}}\right)} \\
& \sim\|f-p\|_{L_{2}\left(\sigma_{j, \mathbf{k}}\right)}+\max _{\mathbf{k}^{\prime} \in \Delta_{j, \mathbf{k}}}\left|\tilde{\lambda}_{j, \mathbf{k}^{\prime}}(f-p)\right|^{2},
\end{aligned}
$$

where we have used that $\left\|\theta_{j, \mathbf{k}}^{\square}\right\|_{L_{2}(\square)} \lesssim 1$. Recall that the functionals $\tilde{\lambda}_{j, \mathbf{k}}^{\square}$ are tensor products of $L_{2}$-inner products and (scaled) point evaluations. Taking the respective scalings into account, it is easy to see that

$$
\left|\tilde{\lambda}_{j, \mathbf{k}^{\prime}}(f)\right| \lesssim 2^{-n j / 2}\|f\|_{L_{\infty}\left(\tilde{\sigma}_{j, \mathbf{k}^{\prime}}\right)},
$$


where $\tilde{\sigma}_{j, \mathbf{k}^{\prime}}:=\operatorname{supp} \tilde{\theta}_{j, \mathbf{k}^{\prime}}$. For $d>n / 2$ the Sobolev embedding theorem ensures that

$$
\|f-p\|_{L_{\infty}(\square)} \lesssim\|f-p\|_{H^{d}(\square)}
$$

while a Bramble-Hilbert type argument yields

$$
\inf _{p \in \Pi_{j, \square}}\|f-p\|_{H^{d}(\square)} \lesssim|f|_{H^{d}(\square)},
$$

where the latter expression is the familiar $d$ th order Sobolev semi-norm. Thus a straightforward scaling argument combined with (3.2.5), (3.2.6) and (3.2.7) implies that for every $\mathbf{k} \in \Delta_{j}^{\square}$ there is a polynomial $p \in \Pi_{j, \square}$ such that

$$
2^{-j n / 2}\|f-p\|_{L_{\infty}\left(\hat{\sigma}_{j, \mathbf{k}}\right)} \lesssim 2^{-d j}|f|_{H^{d}\left(\hat{\sigma}_{j, \mathbf{k}}\right)} .
$$

Bearing the definition of $\Delta_{j, \mathbf{k}}$ and (3.2.3) in mind and substituting the latter estimate into (3.2.4) provides, upon summing over $\mathbf{k} \in \Delta_{j}^{\square}$,

$$
\left\|f-P_{j}^{\square} f\right\|_{L_{2}(\square)}^{2} \lesssim 2^{-2 j d}\|f\|_{H^{d}(\square)},
$$

which completes the proof.

Define in analogy to $(2.4 .1) \lambda_{j, \tilde{\ell}-\tilde{d}}(f):=2^{-j / 2} \tau^{-1} f(0), \lambda_{j, 2^{j}-\mu(d)-\tilde{\ell}+\tilde{d}}(f):=$ $2^{-j / 2} \tau^{-1} f(1)$, where $\tau$ is defined by (2.2.10), and set

$$
\Theta_{j, 0}:=\Theta_{j} \backslash\left\{\theta_{j, \ell-d}, \theta_{j, 2^{j}-\mu(d)-\ell+d}\right\} \text {. }
$$

Then the collections of functionals $\Lambda_{j}:=\left\{\lambda_{j, \tilde{\ell}-\tilde{d}}, \lambda_{j, 2^{j}-\mu(d)-\tilde{\ell}+\tilde{d}}\right\} \cup \Theta_{j, 0}$ satisfy, in view of (2.2.8) and (2.2.9), $\left\langle\tilde{\Theta}_{j}, \Lambda_{j}\right\rangle_{[0,1]}=\mathbf{I}$. Taking again tensor products, the mappings $\tilde{P}_{j}^{\square} f:=\left\langle f, \Lambda_{j}^{\square}\right\rangle_{\square} \tilde{\Theta}_{j}$ are projectors onto the spaces $S\left(\tilde{\Theta}_{j}^{\square}\right)$. Employing the above arguments provides the following facts.

Lemma 3.2.2. The assertions of Lemma 3.2.1 remain true when $\Delta_{j}^{\square}, \partial \Delta_{j}^{\square}, P_{j}^{\square}$, $\tilde{\Lambda}_{j}^{\square}$ and $d$ are replaced by $\tilde{\Delta}_{j}^{\square}, \partial \tilde{\Delta}_{j}^{\square}, \tilde{P}_{j}^{\square}, \Lambda_{j}^{\square}$ and $\tilde{d}$, respectively.

Now let $\tilde{\gamma}:=\sup \left\{s>0: \tilde{\theta} \in H^{s}(\mathbb{R})\right\}$. The following inverse estimates are valid (see e.g. [14, 11]).

Lemma 3.2.3. One has

$$
\left\|v_{j}\right\|_{H^{s}(\square)} \lesssim 2^{j s}\left\|v_{j}\right\|_{L_{2}(\square)}, \quad v_{j} \in V_{j}
$$

provided that

$$
s< \begin{cases}d-1 / 2=: \gamma, & V_{j}=S\left(\Theta_{j}^{\square}\right) \\ \tilde{\gamma}, & V_{j}=S\left(\tilde{\Theta}_{j}^{\square}\right) .\end{cases}
$$

\section{Globally Continuous wavelets on the manifold $\Gamma$}

4.1. Domain decomposition and representation of geometry. Whenever a domain admits a reasonable decomposition into parametric images of cubes we will construct composite wavelet bases on such domains. The following setting covers, in principle, a wide range of bounded domains in Euclidean space as well as closed surfaces embedded in some higher dimensional Euclidean space. In view of boundary integral equations, two-dimensional surfaces in $\mathbb{R}^{3}$ deserve special attention and serve in fact as the primary motivation of the subsequent developments. Nevertheless, open manifolds such as bounded domains in Euclidean space are covered as well. Thus the approach is also suitable for boundary value problems involving second order partial differential operators. 
Throughout the paper the manifold $\Gamma$ will be assumed to be a piecewise smooth manifold (with or without boundary) which is at least globally Lipschitz continuous and endowed with a metric $g$. Denoting the surface element given in local coordinates by $\mathrm{d} s_{x}$, we can define the inner product

$$
\langle u, v\rangle_{\Gamma}:=\int_{\Gamma} u(x) \overline{v(x)} \mathrm{d} s_{x}
$$

for the space $L_{2}(\Gamma)$. An important role in the subsequent development will be played by the modified inner product

$$
\langle f, g\rangle:=\sum_{i=1}^{N}\langle f, g\rangle_{i}, \quad\langle f, g\rangle_{i}:=\left\langle f \circ \kappa_{i}, g \circ \kappa_{i}\right\rangle_{\square},
$$

which is equivalent to $\langle\cdot, \cdot\rangle_{\Gamma}$ in the sense that $\langle\cdot, \cdot\rangle_{\Gamma} \sim\langle\cdot, \cdot\rangle$. In addition to the space $L_{2}(\Gamma)$ we will have to work with Sobolev spaces $H^{s}(\Gamma)$ defined on $\Gamma$. A natural way to define these spaces is to view them as trace spaces. For a certain range of $s$ these spaces can be defined equivalently via an atlas and partitions of unity. We will always assume that we are working in that range. Thus for Lipschitz surfaces we cover $s \leq 1$. Here it is important to note that for closed surfaces one has

$$
H^{s}(\Gamma)^{*}=H^{-s}(\Gamma)
$$

where $H^{s}(\Gamma)^{*}$ is the normed dual of $H^{s}(\Gamma)$.

The construction of $\Psi$ depends crucially on the way the manifold $\Gamma$ is represented. Piecewise defined parametric surface representations appear to be the most practical and rest on the perhaps best developed concepts in computer aided design $(\mathrm{CAD})$. In the sequel we will always assume the following mathematical representation of $\Gamma$. As above we denote by $\square=(0,1)^{n}$ the unit square, which will serve as a fixed parameter domain, and we set

$$
\bar{\Gamma}=\bigcup_{i=1}^{N} \bar{\Gamma}_{i}, \quad \Gamma_{i}=\kappa_{i}(\square), \quad i=1, \ldots, N,
$$

where for some $n \leq n^{\prime}$ the $\kappa_{i}: \mathbb{R}^{n} \rightarrow \mathbb{R}^{n^{\prime}}$ are smooth functions chosen in such a way that $\Gamma$ has the desired degree of global smoothness. Note that the patches $\Gamma_{i}$ are not supposed to overlap:

$$
\Gamma_{i} \cap \Gamma_{j}=\emptyset, \quad i \neq j,
$$

i.e., the different patches do not intersect. However, we do require that the partition (4.1.3) is conforming in the sense that the intersection of any two patches $\bar{\Gamma}_{i}, \bar{\Gamma}_{l}$ is either empty or a common lower dimensional face. $\Gamma$ is said to be a $C^{m}$-surface if there exist local $C^{m}$ reparametrizations, i.e., for any neighborhood $\mathcal{N} \subseteq \Gamma$, say, such that $\mathcal{N} \cap \Gamma_{i} \neq \emptyset, \mathcal{N} \cap \Gamma_{j} \neq \emptyset$, there exist an affine map $\sigma$, a neighborhood $\mathcal{M} \subseteq \square \cup \sigma(\square)$ and a function $\kappa \in C^{m}(\mathcal{M})$ such that

$$
\left.\kappa\right|_{\square \cap \mathcal{M}}=\kappa_{i},\left.\quad \kappa\right|_{\sigma(\square) \cap \mathcal{M}}=\kappa_{j} \circ \rho \circ \sigma^{-1},
$$

where $\rho$ is some regular reparametrization of $\sigma(\square)$. In practical realizations for most of the patches the local reparametrizations $\rho$ can be chosen as the identity, which means that the parametrizations join in such a way that their coordinate functions are $C^{m}$. In the case $m=0$ we will always assume that $\Gamma$ is a Lipschitz manifold. The only places where nontrivial reparametrizations have to be employed is near singular vertices, by which we mean vertices sharing a number of patches which is different from $2^{n}$. Depending on the genus of the surface, such singular 
vertices may always have to occur. We also remark that in all practical realizations the parametrizations $\kappa_{i}$ are actually polynomial or piecewise polynomial with sufficiently high componentwise smoothness.

We have chosen this setting since a variety of practical tools have been developed in the CAD community realizing such surfaces for an essentially arbitrary topology. Specifically, in $[20,24]$ a practicable concept for generating $C^{0}, C^{1}$ and $C^{2}$ surfaces of arbitrary topology is developed employing only quadrilateral patches, as required above. Moreover, these surfaces can be refined by means of subdivisions, which therefore fits into the present context. Thus we may view a surface $\Gamma$ of the above type as the true target surface, or as an approximation which could be successively improved if necessary.

4.2. Globally continuous biorthogonal multiresolution on $\Gamma$. It is now straightforward to construct wavelet bases on $\Gamma$ by parametric lifting of the bases on $\square$ to each patch $\Gamma_{i}$. The resulting functions are, of course, only continuous in each patch, so that the corresponding bases on $\Gamma$ are suitable for zero order operators. Note that the cancellation properties on $\square$ are naturally preserved. An advantage over (discontinuous) multi-wavelets is that, due to the smoothness inside each patch, the space dimensions increase less rapidly with the order of the trial spaces. However, when dealing e.g. with conforming methods for second order differential operators, the trial spaces and their multiscale decompositions should be continuous over $\Gamma$. We will concentrate on this case for the remainder of this paper.

The following way of joining adjacent multiresolution bases continuously makes essential use of the following consequence of the symmetry relations in (2.1.9) and the properties listed in Remark 2.4.3.

Remark 4.2.1. Suppose that $\rho$ is an affine mapping of $\square$ onto itself. Then

$$
\Theta_{j}^{\square} \circ \rho=\Theta_{j}^{\square}, \quad \tilde{\Theta}_{j}^{\square} \circ \rho=\tilde{\Theta}_{j}^{\square} .
$$

In order to organize patching the local spaces together, it is convenient to work with a geometrical grid that reflects topological connectivities. To this end, consider (again for the univariate case) the mapping $q_{j}$ defined on $\Delta_{j}=\Delta_{j}^{L} \cup \Delta_{j}^{I} \cup \Delta_{j}^{R}$ (see $(2.1 .5),(2.1 .8))$ by

$$
q_{j}(k):= \begin{cases}0, & k=\ell-d, \\ 1, & k=2^{j}-\mu(d)-\ell+d, \\ 2^{-j} k, & k \neq \ell-d, 2^{j}-\mu(d)-\ell+d\end{cases}
$$

(i.e., the first and last index are identified with the end points of the interval), and set

$$
\mathbf{q}_{j}(\mathbf{k}):=\left(q\left(k_{1}\right), \ldots, q\left(k_{n}\right)\right), \quad \mathbf{k} \in \Delta_{j}^{\square} .
$$

Moreover, let the physical local grids, their 'interiors' and the physical global grid be defined by

$$
\square_{j, i}:=\kappa_{i}\left(\mathbf{q}_{j}\left(\Delta_{j}^{\square}\right)\right), \quad \square_{j, i}^{0}:=\kappa_{i}\left(\mathbf{q}_{j}\left(\Delta_{j}^{\square} \backslash \partial \Delta_{j}^{\square}\right)\right), \quad \square_{j}:=\bigcup_{i=1}^{N} \square_{j, i} .
$$

The local 'grid boundaries' and the 'discrete skeleton' are given by

$$
\partial \square_{j, i}:=\square_{j, i} \backslash \square_{j, i}^{0}, \quad \partial \square_{j}:=\bigcup_{i=1}^{N} \partial \square_{j, i} .
$$


Of course, points which geometrically coincide will be identified. Thus $\square_{j}, \square_{j, i}, \square_{j, i}^{0}$, $\partial \square_{j, i}^{0}$ stand for all grid points on $\Gamma$, the grid points on patch $\Gamma_{i}$, the grid points in the interior of patch $\Gamma_{i}$ and the grid points located on the faces of the patches $\Gamma_{i}$, respectively. Each grid point in $\square_{j}$ is identified by the patch number $i$ and the corresponding parametric image of the point $\mathbf{q}_{j}(\mathbf{k}), \mathbf{k} \in \Delta_{j}^{\square}$. We collect this information in the index $\xi=\xi(i, \mathbf{k})=\kappa_{i}\left(\mathbf{q}_{j}(\mathbf{k})\right)$. For $\xi \in \square_{j} \backslash \partial \square_{j}$ the representation is unique. However, $\xi \in \partial \square_{j}$ may have several representations

$$
\xi=\kappa_{i_{1}}\left(\mathbf{q}_{j}\left(\mathbf{k}^{1}\right)\right)=\cdots=\kappa_{i_{r}}\left(\mathbf{q}_{j}\left(\mathbf{k}^{r}\right)\right),
$$

where $r(\xi)$ is the number of patches $\overline{\Gamma_{i}}$ containing $\xi$. We will always refer to this representation, where of course $r(\xi)=1$ if $\xi \in \square_{j} \backslash \partial \square_{j}$.

For the simple case $\Gamma=[0,1] \cup[1,2]$ Figure 3 illustrates the glueing of generator basis functions across the interface $\{1\}$. This carries over to the multivariate case in a straightforward manner. In fact, with the above conventions in mind we define, for $\xi \in \bigcap_{l=1}^{r(\xi)} \bar{\Gamma}_{i_{l}}$,

$$
\phi_{j, \xi}(\mathbf{x}):=\theta_{j, \mathbf{k}^{l}}^{\square}\left(\kappa_{i_{l}}^{-1}(\mathbf{x})\right), \quad \mathbf{x} \in \Gamma_{i_{l}}, l=1, \ldots, r(\xi),
$$

i.e., the restriction of $\phi_{j, \xi}$ to any patch $\Gamma_{i_{l}}$ containing $\xi$ is a parametric lifting of a tensor product generator involving corresponding factors of boundary adapted univariate generators. Except for normalization, the $\tilde{\phi}_{j, \xi}$ are defined analogously by

$$
\tilde{\phi}_{j, \xi}(\mathbf{x}):=\frac{1}{r(\xi)} \tilde{\theta}_{j, \mathbf{k}^{l}}^{\square}\left(\kappa_{i_{l}}^{-1}(\mathbf{x})\right), \quad \mathbf{x} \in \Gamma_{i_{l}}, l=1, \ldots, r(\xi) .
$$

In view of the boundary conditions $(2.2 .8),(2.2 .9)$, it is clear that $\phi_{j, \xi}, \tilde{\phi}_{j, \xi}$ are fully supported in a single patch $\bar{\Gamma}_{i}$ if $\xi \in \square_{j, i}^{0}$ for some $i(r(\xi)=1)$, and hence are continuous on $\Gamma$. By Remark 4.2.1, also those $\phi_{j, \xi}, \tilde{\phi}_{j, \xi}$ with $\xi \in \partial \square_{j}$, whose supports intersect $r(\xi)$ patches, are continuous, and the normalization ensures that the sets

$$
\Phi_{j}:=\left\{\phi_{j, \xi}: \xi \in \square_{j}\right\}, \quad \tilde{\Phi}_{j}:=\left\{\tilde{\phi}_{j, \xi}: \xi \in \square_{j}\right\},
$$

are still biorthogonal:

$$
\left\langle\Phi_{j}, \tilde{\Phi}_{j}\right\rangle=\mathbf{I}
$$
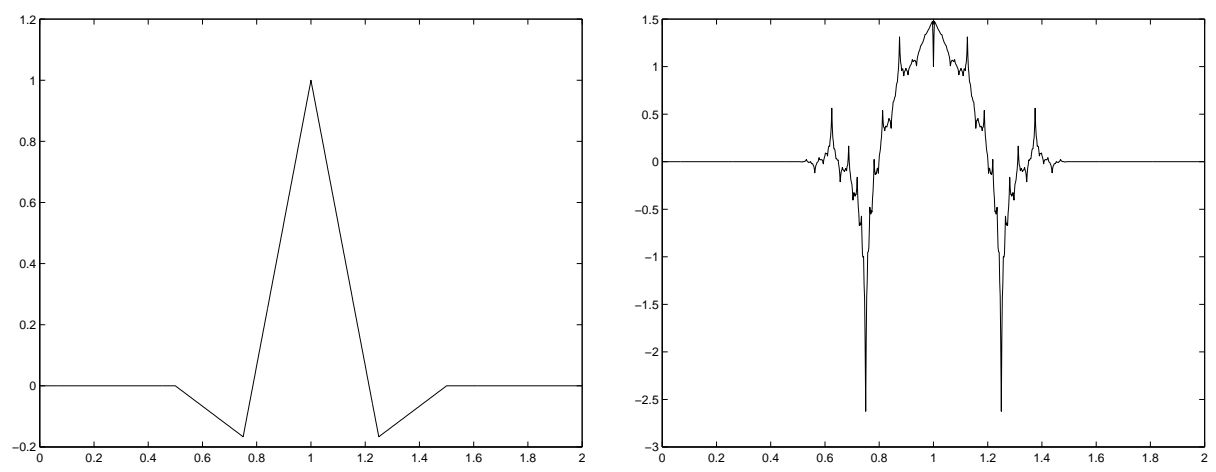

FiguRE 3 . Generators $\phi_{j, \xi}$ and $\tilde{\phi}_{j, \xi}, d=\tilde{d}=2$ across the interface $\{\xi=1\}$ 
where $\langle\cdot, \cdot\rangle$ is defined by (4.1.2). Moreover, invoking for instance Lemma 2.1 in [14], we immediately confirm the uniform stability

$$
\|\mathbf{c}\|_{\ell_{2}\left(\square_{j}\right)} \sim\left\|\mathbf{c}^{T} \Phi_{j}\right\|_{L_{2}(\Gamma)} \sim\left\|\mathbf{c}^{T} \tilde{\Phi}_{j}\right\|_{L_{2}(\Gamma)}
$$

of the generator bases $\Phi_{j}, \tilde{\Phi}_{j}$.

4.3. Refinement relations. The bases $\Phi_{j}, \tilde{\Phi}_{j}$ are, of course, still refinable. We will briefly identify next the corresponding refinement matrices $\mathbf{M}_{j, \mathbb{Q}}^{\Gamma}, \tilde{\mathbf{M}}_{j, \mathbb{Q}}^{\Gamma}$ satisfying

$$
\Phi_{j}^{T}=\Phi_{j+1}^{T} \mathbf{M}_{j, 0}^{\Gamma}, \quad \tilde{\Phi}_{j}^{T}=\tilde{\Phi}_{j+1}^{T} \tilde{\mathbf{M}}_{j, 0}^{\Gamma} .
$$

Proposition 4.3.1. For the matrices $\mathbf{M}_{j, \mathbb{0}}^{\Gamma}, \tilde{\mathbf{M}}_{j, \mathbb{Q}}^{\Gamma}$ in (4.3.1) one has that the inequality $\left(\tilde{\mathbf{M}}_{j, \mathbb{O}}^{\Gamma}\right)_{\zeta, \xi},\left(\mathbf{M}_{j, \mathbb{O}}^{\Gamma}\right)_{\zeta, \xi} \neq 0$ can only occur when there exists an $i$ such that both $\zeta$ and $\xi$ belong to (the closed patch) $\bar{\Gamma}_{i}$, i.e.,

$$
\left(\mathbf{M}_{j, 0}^{\Gamma}\right)_{\zeta, \xi}=\left(\tilde{\mathbf{M}}_{j, 0}^{\Gamma}\right)_{\zeta, \xi}=0 \text {, when } \zeta \cap \xi=\emptyset,
$$

where $\xi \cap \zeta=\emptyset$ means that there exists no $i$ such that both $\xi$ and $\zeta$ belong to $\bar{\Gamma}_{i}$. Moreover, for $\zeta, \xi \in \bar{\Gamma}_{i}$, i.e., $\zeta=\kappa_{i}\left(\mathbf{q}_{j+1}\left(\mathbf{k}^{\prime}\right)\right) \in \square_{j+1, i}, \xi=\kappa_{i}\left(\mathbf{q}_{j}(\mathbf{k})\right) \in \square_{j, i}$, one has

$$
\left(\mathbf{M}_{j, \mathbb{0}}^{\Gamma}\right)_{\zeta, \xi}:=\left(\mathbf{M}_{j, 0}^{\square}\right)_{\mathbf{k}^{\prime}, \mathbf{k}}, \quad\left(\tilde{\mathbf{M}}_{j, \mathbb{0}}^{\Gamma}\right)_{\zeta, \xi}:=\frac{r(\zeta)}{r(\xi)}\left(\tilde{\mathbf{M}}_{j, 0}^{\square}\right)_{\mathbf{k}^{\prime}, \mathbf{k}} .
$$

Proof. (4.3.2) follows directly from the definitions (4.2.4), (4.2.5) and (2.3.3) and Remark 2.4.3.

As for (4.3.3), let $\xi=\kappa_{i}\left(\mathbf{q}_{j}(\mathbf{k})\right) \in \square_{j, i}$. Then for $\mathbf{x} \in \Gamma_{i}$ one has

$$
\begin{aligned}
\phi_{j, \xi}(\mathbf{x}) & =\theta_{j, \mathbf{k}}^{\square}\left(\kappa_{i}^{-1}(\mathbf{x})\right)=\sum_{\mathbf{k}^{\prime} \in \Delta_{j+1}^{\square}}\left(\mathbf{M}_{j, 0}^{\square}\right)_{\mathbf{k}^{\prime}, \mathbf{k}} \theta_{j+1, \mathbf{k}^{\prime}}^{\square}\left(\kappa_{i}^{-1}(\mathbf{x})\right) \\
& =\sum_{\mathbf{k}^{\prime} \in \Delta_{j+1}^{\square}}\left(\mathbf{M}_{j, 0}^{\square}\right)_{\mathbf{k}^{\prime}, \mathbf{k}} \phi_{j+1, \kappa_{i}\left(\mathbf{q}_{j+1}\left(\mathbf{k}^{\prime}\right)\right)}(\mathbf{x}) .
\end{aligned}
$$

So it only remains to confirm that (4.3.3) is consistent when $\zeta$ and $\xi$ both belong to the same patch boundary. On account of (4.1.5) and (2.3.2) this is indeed the case, which finishes the proof for $\mathbf{M}_{j, 0}^{\Gamma}$. The same reasoning works for $\tilde{\phi}_{j, \xi}$ when $\xi \in \square_{j, i}^{0}$. Now suppose that $\xi \in \partial \square_{j, i}$ satisfies (4.2.3). Then for $\mathbf{x} \in \bar{\Gamma}_{i_{l}}$ one has

$$
\begin{aligned}
\tilde{\phi}_{j, \xi}(\mathbf{x}) & =\frac{1}{r(\xi)} \tilde{\theta}_{j, \mathbf{k}^{l}}^{\square}\left(\kappa_{i_{l}}^{-1}(\mathbf{x})\right)=\frac{1}{r(\xi)} \sum_{\mathbf{k}^{\prime} \in \Delta_{j+1}^{\square}}\left(\tilde{\mathbf{M}}_{j, \mathbb{0}}^{\square}\right)_{\mathbf{k}^{\prime}, \mathbf{k}^{l}} \tilde{\theta}_{j+1, \mathbf{k}^{\prime}}^{\square}\left(\kappa_{i_{l}}^{-1}(\mathbf{x})\right) \\
& =\sum_{\mathbf{k}^{\prime} \in \Delta_{j+1}^{\square}} \frac{r\left(\kappa_{i_{l}}\left(\mathbf{q}_{j+1}\left(\mathbf{k}^{\prime}\right)\right)\right)}{r(\xi)}\left(\tilde{\mathbf{M}}_{j, \mathbb{O}}^{\square}\right)_{\mathbf{k}^{\prime}, \mathbf{k}} \tilde{\phi}_{j+1, \kappa_{i_{l}}\left(\mathbf{q}_{j+1}\left(\mathbf{k}^{\prime}\right)\right)}(\mathbf{x}),
\end{aligned}
$$

which finishes the proof.

4.4. Stable completions and wavelets. Our objective now is to construct complement bases $\Psi_{j}, \tilde{\Psi}_{j}$ which are stable in the sense of (2.2.7) or (4.2.7) such that in addition the biorthogonality relations

$$
S\left(\Phi_{j+1}\right)=S\left(\Phi_{j}\right) \oplus S\left(\Psi_{j}^{\Gamma}\right), \quad S\left(\tilde{\Phi}_{j+1}\right)=S\left(\tilde{\Phi}_{j}\right) \oplus S\left(\tilde{\Psi}_{j}^{\Gamma}\right)
$$

and

$$
S\left(\tilde{\Psi}_{j}^{\Gamma}\right) \perp S\left(\Phi_{j}\right), \quad S\left(\Psi_{j}^{\Gamma}\right) \perp S\left(\tilde{\Phi}_{j}\right)
$$


hold, i.e., the union of the $\Psi_{j}^{\Gamma}$ (respectively $\tilde{\Psi}_{j}^{\Gamma}$ ) over all levels $j$ are candidates for biorthogonal wavelet bases. This is known to be necessary for the validity of norm equivalences including $L_{2}$ [10]. The construction will proceed in two steps. First we will identify some 'easily available' initial complement basis $\breve{\Psi}_{j}^{\Gamma}$, which will then be modified into the desired one. The main vehicle is the observation that space decompositions of the above type are equivalent to finding some $\# \square_{j+1} \times\left(\# \square_{j+1}-\right.$ $\left.\# \square_{j}\right)$ matrix $\breve{\mathbf{M}}_{j, 1}^{\Gamma}$ such that the composed matrix $\breve{\mathbf{M}}_{j}^{\Gamma}:=\left(\mathbf{M}_{j, \mathbb{0}}^{\Gamma}, \breve{\mathbf{M}}_{j, 1}^{\Gamma}\right)$ is invertible and the spectral norms of $\breve{\mathbf{M}}_{j}^{\Gamma}$ and $\breve{\mathbf{G}}_{j}^{\Gamma}=\left(\begin{array}{c}\breve{\mathbf{G}}_{j, 0}^{\Gamma} \\ \breve{\mathbf{G}}_{j, 1}^{\Gamma}\end{array}\right):=\left(\breve{\mathbf{M}}_{j}^{\Gamma}\right)^{-1}$ are uniformly bounded [5]. Matrices $\breve{\mathbf{M}}_{j, 1}^{\Gamma}$ with this property are called stable completions of $\mathbf{M}_{j, \mathbb{0}}^{\Gamma}$.

To describe first appropriate index sets for the wavelets on $\Gamma$, recall that $\nabla_{j}:=$ $\left\{1 / 2, \ldots, 2^{j}-1 / 2\right\}$ denote the index sets for the univariate complement basis functions on the interval $[0,1]$. The structure of the index sets for the wavelet bases $\Psi_{j}^{\square}$ looks then as follows:

$$
\nabla_{j}^{\square}:=\bigcup_{\mathbf{e} \in\{0,1\}^{n} \backslash \mathbb{0}} \nabla_{j, \mathbf{e}}^{\square}
$$

where

$$
\nabla_{j, \mathbf{e}}^{\square}:=\chi_{l=1}^{n} \nabla_{j, e_{l}}, \quad \nabla_{j, e_{l}}:=\left\{\begin{array}{cc}
\Delta_{j}, & e_{l}=0 \\
\nabla_{j}, & e_{l}=1 .
\end{array}\right.
$$

As before we will associate the elements of $\nabla_{j}^{\square}$ with grid points in the patches $\Gamma_{i}$. Define

by

$$
\mathbf{w}_{j}(\mathbf{k}, \mathbf{e}):=\left(w_{j}\left(k_{1}, e_{1}\right) \ldots w_{j}\left(k_{n}, e_{n}\right)\right), \quad \mathbf{k} \in \nabla_{j, \mathbf{e}}^{\square}
$$

$$
w_{j}\left(k_{l}, e_{l}\right):= \begin{cases}q_{j}\left(k_{l}\right), & e_{l}=0, k_{l} \in \Delta_{j} \\ 2^{-j} k_{l}, & e_{l}=1, k_{l} \in \nabla_{j}\end{cases}
$$

This gives rise to the grids

$$
\nabla_{j, i, \mathbf{e}}:=\kappa_{i}\left(\mathbf{w}_{j}\left(\nabla_{j, \mathbf{e}}^{\square}, \mathbf{e}\right)\right), \quad \nabla_{j}^{\Gamma}:=\bigcup_{i=1}^{N} \bigcup_{\mathbf{e} \in\{0,1\}^{n} \backslash\{0\}} \nabla_{j, i, \mathbf{e}} .
$$

We are now prepared to formulate the central ingredient of the construction, namely to identify a suitable stable completion for the refinement matrices $\mathbf{M}_{j, 0}^{\Gamma}$ described in Proposition 4.3.1 with the aid of local univariate stable completions of the refinement matrices $\mathbf{M}_{j, 0}$ corresponding to generator bases on the interval.

Theorem 4.4.1. Let $\breve{\mathbf{M}}_{j, 1}$ be any (univariate) stable completion of $\mathbf{M}_{j, 0}=: \breve{\mathbf{M}}_{j, 0}$ (on $[0,1])$ with the following properties:

(i) For the corresponding inverse blocks $\breve{\mathbf{G}}_{j, 0}, \breve{\mathbf{G}}_{j, 1}$ one has

$$
\breve{\mathbf{M}}_{j}^{\uparrow}=\breve{\mathbf{M}}_{j}, \quad \breve{\mathbf{G}}_{j}^{\uparrow}=\breve{\mathbf{G}}_{j},
$$

(see Proposition 2.3.1 and Remark 2.4.2).

(ii) The first and last row of $\breve{\mathbf{M}}_{j, 1}$ have only zero entries:

$$
\left(\breve{\mathbf{M}}_{j, 1}\right)_{k, k^{\prime}}=0, \quad k \in\left\{\ell-d, 2^{j+1}-\mu(d)-\ell+d\right\}, k^{\prime} \in \nabla_{j}
$$

(see (2.1.8)). 
(iii) The $\breve{\mathbf{M}}_{j, 1}$ are uniformly sparse, i.e., the number of nonzero entries and their modulus in the rows and columns of $\breve{\mathbf{M}}_{j, 1}$ remain uniformly bounded.

Let $\breve{\mathbf{M}}_{j, \mathbf{e}}^{\square}$ and $\breve{\mathbf{G}}_{j, \mathbf{e}}^{\square}$ be defined according to (3.1.1). Moreover, let

$$
c(\nu, \xi):=\#\left\{i: \xi \in \bar{\Gamma}_{i} \wedge \nu \in \bar{\Gamma}_{i}\right\},
$$

and define, for $\zeta=\kappa_{i}\left(\mathbf{q}_{j+1}\left(\mathbf{k}^{\prime}\right)\right), \xi \in \kappa_{i}\left(\mathbf{w}_{j}(\mathbf{k}, \mathbf{e})\right)$,

$$
\left(\breve{\mathbf{M}}_{j, \mathbf{e}}^{\Gamma}\right)_{\zeta, \xi}:=\left(\breve{\mathbf{M}}_{j, \mathbf{e}}^{\square}\right)_{\mathbf{k}^{\prime}, \mathbf{k}}, \quad\left(\breve{\mathbf{G}}_{j, \mathbf{e}}^{\Gamma}\right)_{\xi, \zeta}:=\frac{c(\xi, \zeta)}{r(\xi)}\left(\breve{\mathbf{G}}_{j, \mathbf{e}}^{\square}\right)_{\mathbf{k}, \mathbf{k}^{\prime}}, \quad \mathbf{e} \in\{0,1\}^{n},
$$

while we let

$$
\left(\breve{\mathbf{M}}_{j, \mathbf{e}}^{\Gamma}\right)_{\zeta, \xi}=\left(\breve{\mathbf{G}}_{j, \mathbf{e}}^{\Gamma}\right)_{\xi, \zeta}:=0 \text {, if } \xi \cap \zeta=\emptyset .
$$

Then $\breve{\mathbf{M}}_{j, 1}^{\Gamma}:=\left(\breve{\mathbf{M}}_{j, \mathbf{e}}^{\Gamma}\right)_{\mathbf{e} \in\{0,1\} \backslash\{0\}}$ is a stable completion for $\mathbf{M}_{j, \mathbb{0}}^{\Gamma}$, and

$$
\breve{\mathbf{G}}_{j, \mathbf{e}}^{\Gamma} \breve{\mathbf{M}}_{j, \mathbf{e}^{\prime}}^{\Gamma}=\delta_{\mathbf{e}, \mathbf{e}^{\prime}} \mathbf{I}, \quad \mathbf{e}, \mathbf{e}^{\prime} \in\{0,1\}^{n} .
$$

Proof. In view of (4.4.5), the consistency of the definitions (4.4.8) follows by the same reasoning as before for the refinement matrices. To verify (4.4.10), suppose that

$$
\nu=\kappa_{i_{1}}\left(\mathbf{w}_{j}\left(\mathbf{k}^{1}, \mathbf{e}\right)\right)=\cdots=\kappa_{i_{r(\nu)}}\left(\mathbf{w}_{j}\left(\mathbf{k}^{r(\nu)}, \mathbf{e}\right)\right),
$$

and consider the entry

$$
\Sigma_{\nu, \mu}^{\mathbf{e}, \mathbf{e}^{\prime}}:=\sum_{\xi \in \square_{j+1}}\left(\breve{\mathbf{G}}_{j, \mathbf{e}}^{\Gamma}\right)_{\nu, \xi}\left(\breve{\mathbf{M}}_{j, \mathbf{e}^{\prime}}^{\Gamma}\right)_{\xi, \mu}
$$

of the product in question. We wish to break the sum over $\square_{j+1}$ into sums over the local grids $\square_{j+1, i}$. By (4.4.9) the factor $\left(\breve{G}_{j, \mathbf{e}}^{\Gamma}\right)_{\nu, \xi}$ can only differ from zero if $\xi \in \square_{j+1, i_{l}}, l=1, \ldots, r(\nu)$. Likewise, for any $\xi \in \square_{j+1, i_{l}}$ the factor $\left(\breve{\mathbf{M}}_{j, \mathbf{e}^{\prime}}^{\Gamma}\right)_{\xi, \mu}$ can only be nonzero if there exists $i^{\prime}$ such that $\xi$ and $\mu$ both belong to $\bar{\Gamma}_{i^{\prime}}$. Suppose first that $i^{\prime} \neq i_{l}$. Then $\xi$ must belong to an interface $\square_{j+1, i_{l}} \cap \square_{j+1, i^{\prime}}$, whereas $\mu$ does not lie on that interface. However, then

$$
\left(\breve{\mathbf{M}}_{j, \mathbf{e}^{\prime}}^{\Gamma}\right)_{\xi, \mu}=\left(\breve{\mathbf{M}}_{j, \mathbf{e}^{\prime}}^{\Gamma}\right)_{\kappa_{i^{\prime}}\left(\mathbf{q}_{j+1}\left(\overline{\mathbf{k}}^{\prime}\right)\right), \kappa_{i^{\prime}}\left(\mathbf{w}_{j}\left(\overline{\mathbf{k}}, \mathbf{e}^{\prime}\right)\right)}=\left(\breve{\mathbf{M}}_{j, \mathbf{e}^{\prime}}^{\square}\right)_{\overline{\mathbf{k}}^{\prime}, \overline{\mathbf{k}}}
$$

has a factor $\left(\breve{\mathbf{M}}_{j, e_{p}^{\prime}}\right)_{\bar{k}_{p}^{\prime}, \bar{k}_{p}}$, where $\bar{k}_{p}^{\prime}$ is a boundary index while $\bar{k}_{p}$ is not, so that (2.3.3) and the assumption (4.4.6) imply that this factor vanishes. Hence

$$
G(\nu, \mu):=\left\{i \in\left\{i_{1}, \ldots, i_{r(\nu)}\right\}: \mu \in \bar{\Gamma}_{i}\right\}
$$

selects those local grids $\square_{j+1, i}$ that could contribute nonzero summands to $\Sigma_{\nu, \mu}^{\mathbf{e}, \mathbf{e}^{\prime}}$. However, when summing over each such $\square_{j+1, i}$ some interfaces may be visited several times, so these summands have to be properly weighted. In fact, setting

$$
c(\nu, \xi, \mu):=\#\left\{i: \nu, \xi, \mu \in \bar{\Gamma}_{i}\right\},
$$

the quantity $\Sigma_{\nu, \mu}^{\mathbf{e}, \mathbf{e}^{\prime}}$ reduces, on account of (4.4.8), to

$$
\begin{array}{r}
\sum_{i \in G(\nu, \mu)} \sum_{\mathbf{k}^{\prime} \in \square_{j+1, i}} \frac{c\left(\nu, \kappa_{i}\left(\mathbf{q}_{j+1}\left(\mathbf{k}^{\prime}\right)\right)\right.}{r(\nu) c\left(\kappa_{i}\left(\mathbf{w}_{j}\left(\mathbf{k}^{i}, \mathbf{e}\right)\right), \kappa_{i}\left(\mathbf{q}_{j+1}\left(\mathbf{k}^{\prime}\right)\right), \kappa_{i}\left(\mathbf{w}_{j}\left(\overline{\mathbf{k}}^{i}, \mathbf{e}^{\prime}\right)\right)\right)} \\
\times\left(\breve{\mathbf{G}}_{j, \mathbf{e}}^{\square}\right)_{\mathbf{k}^{i}, \mathbf{k}^{\prime}}\left(\breve{\mathbf{M}}_{j, \mathbf{e}^{\prime}}^{\square}\right)_{\mathbf{k}^{\prime}, \overline{\mathbf{k}}^{i}} .
\end{array}
$$


We will show next that

$$
c(\nu, \xi, \mu)=c(\nu, \xi) \text { or }\left(\breve{\mathbf{G}}_{j, \mathbf{e}}^{\square}\right)_{\mathbf{k}^{i}, \mathbf{k}^{\prime}}\left(\breve{\mathbf{M}}_{j, \mathbf{e}^{\prime}}^{\square}\right)_{\mathbf{k}^{\prime}, \overline{\mathbf{k}}^{i}}=0 .
$$

Obviously $c(\nu, \xi, \mu) \leq c(\nu, \xi)$. Suppose that for some $\xi$ strict inequality $c(\nu, \xi, \mu)<$ $c(\nu, \xi)$ holds. This means that $\xi$ and $\nu$ belong to some interface which does not contain $\mu$. Hence $\left(\breve{\mathbf{M}}_{j, \mathbf{e}^{\prime}}^{\square}\right)_{\mathbf{k}^{\prime}, \overline{\mathbf{k}}^{i}}$ contains a factor $\left(\breve{\mathbf{M}}_{j, e_{p}^{\prime}}\right)_{k_{p}^{\prime}, \bar{k}_{p}^{i}}$ such that $k_{p}^{\prime}$ is a boundary index (referring to one of the two generator basis functions in $\Theta_{j+1}$ that do not vanish at zero and one), whereas $\bar{k}_{p}^{i}$ refers either to an element in $\breve{\Omega}_{j}$ or to a basis function in $\Theta_{j}$ whose index does not belong to the end points of [0,1]. By assumption (4.4.6) and the nature of the refinement equations for $\Theta_{j}$ (see [14]), in either case these functions vanish at zero and one. Therefore the two-scale relation does not involve an element of $\Theta_{j+1}$ that does not vanish at zero or one, which means that $\left(\breve{M}_{j, e_{p}^{\prime}}\right)_{k_{p}^{\prime}, \bar{k}_{p}^{i}}=0$. This confirms (4.4.13). Since for all nonzero summands in $\Sigma_{\nu, \mu}^{\mathbf{e}, \mathbf{e}^{\prime}}$ therefore $c(\nu, \xi)=c(\nu, \xi, \mu)$, we obtain from (4.4.12)

$$
\Sigma_{\nu, \mu}^{\mathbf{e}, \mathbf{e}^{\prime}}=\frac{1}{r(\nu)} \sum_{i \in G(\nu, \mu)} \sum_{\mathbf{k}^{\prime} \in \square_{j+1, i}}\left(\breve{\mathbf{G}}_{j, \mathbf{e}}^{\square}\right)_{\mathbf{k}^{i}, \mathbf{k}^{\prime}}\left(\breve{\mathbf{M}}_{j, \mathbf{e}^{\prime}}^{\square}\right)_{\mathbf{k}^{\prime}, \overline{\mathbf{k}}^{i}} .
$$

Since obviously by (3.1.1) $\breve{\mathbf{G}}_{j} \breve{\mathbf{M}}_{j}=\mathbf{I}$ implies $\breve{\mathbf{G}}_{j, \mathbf{e}}^{\square} \breve{\mathbf{M}}_{j, \mathbf{e}^{\prime}}^{\square}=\delta_{\mathbf{e}, \mathbf{e}^{\prime}} \mathbf{I}$, one has

$$
\sum_{\mathbf{k}^{\prime} \in \square_{j+1, i}}\left(\breve{\mathbf{G}}_{j, \mathbf{e}}^{\square}\right)_{\mathbf{k}^{i}, \mathbf{k}^{\prime}}\left(\breve{\mathbf{M}}_{j, \mathbf{e}^{\prime}}^{\square}\right)_{\mathbf{k}^{\prime}, \overline{\mathbf{k}}^{i}}=\delta_{\mathbf{e}, \mathbf{e}^{\prime}} \delta_{\mathbf{k}^{i}, \overline{\mathbf{k}}^{i}}
$$

so that

$$
\Sigma_{\nu, \mu}^{\mathbf{e}, \mathbf{e}^{\prime}}=\frac{1}{r(\nu)} \sum_{i \in G(\nu, \mu)} \delta_{\mathbf{e}, \mathbf{e}^{\prime}} \delta_{\mathbf{k}^{i}, \overline{\mathbf{k}}^{i}}
$$

But when $\mathbf{k}^{i}=\overline{\mathbf{k}}^{i}$ one clearly has $\# G(\nu, \mu)=\# G(\nu, \nu)=r(\nu)$, which confirms (4.4.10).

By construction, each row and each column of the matrices $\breve{\mathbf{M}}_{j}^{\Gamma}, \breve{\mathbf{G}}_{j}^{\Gamma}$ contains only finitely many nonzero entries, whose number and modulus is uniformly bounded in the level $j$. Hence their spectral norms are uniformly bounded. By Corollary 2.1 in [5], this implies the stability of the completions $\breve{\mathbf{M}}_{j, 1}^{\Gamma}=\left(\breve{\mathbf{M}}_{j, \mathbf{e}}^{\Gamma}\right)_{\mathbf{e} \in\{0,1\}^{n} \backslash\{\mathbb{0}\}}$, which completes the proof.

Recall from Remark 2.4.3 that the stable completions $\breve{\mathbf{M}}_{j, 1}$ constructed in Remark 2.4.2 satisfy the hypotheses in Theorem 4.4.1.

There is some flexibility in choosing $\breve{\mathbf{M}}_{j, 1}$ in Theorem 4.4.1. A particularly convenient choice for bilinear trial functions $d=2$ is the nodal hierarchical basis employed in the examples shown in Figure 4.

The functions in $\left(\breve{\Psi}_{j}^{\Gamma}\right)^{T}:=\Phi_{j+1}^{T} \breve{\mathbf{M}}_{j, 1}^{\Gamma}$ form (uniformly) stable bases for certain complements of $S\left(\Phi_{j}\right)$ in $S\left(\Phi_{j+1}\right)$, but they do not yet form a biorthogonal basis over all levels, which is needed for the Riesz basis property.

However, we are now ready to construct stable completions corresponding to biorthogonal wavelets on $\Gamma$. The main ingredients are the explicit initial stable completions $\breve{\mathbf{M}}_{j, 1}^{\Gamma}$ constructed in Theorem 4.4.1. 

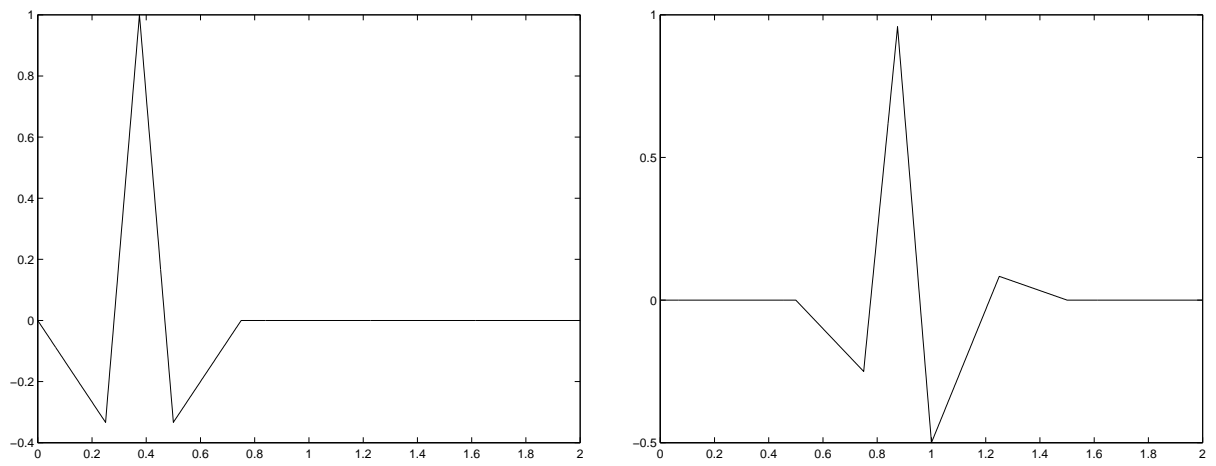

Figure 4. Primal wavelets $\psi_{j, 3 \cdot 2^{-j-1}}^{\Gamma}$ and $\psi_{j, \xi-2^{-j-1}}^{\Gamma} d=\tilde{d}=2$ at the interface $\{\xi=1\}$

Theorem 4.4.2. For $\breve{\mathbf{M}}_{j}^{\Gamma}, \breve{\mathbf{G}}_{j}^{\Gamma}$ from Theorem 4.4.1 let

$$
\mathbf{M}_{j, 1}^{\Gamma}:=\left(\mathbf{I}-\mathbf{M}_{j, \mathbb{O}}^{\Gamma}\left(\tilde{\mathbf{M}}_{j, \mathbb{Q}}^{\Gamma}\right)^{T}\right) \breve{\mathbf{M}}_{j, 1}^{\Gamma} .
$$

Then the inverse of $\mathbf{M}_{j}^{\Gamma}=\left(\mathbf{M}_{j, 0}^{\Gamma}, \mathbf{M}_{j, 1}^{\Gamma}\right)$ is given by

$$
\mathbf{G}_{j}^{\Gamma}=\left(\begin{array}{c}
\left(\tilde{\mathbf{M}}_{j, 0}^{\Gamma}\right)^{T} \\
\breve{G}_{j, 1}^{\Gamma}
\end{array}\right) .
$$

Moreover, the collections

$$
\Psi_{j}^{\Gamma}:=\left(\mathbf{M}_{j, 1}^{\Gamma}\right)^{T} \Phi_{j+1}, \quad \tilde{\Psi}_{j}^{\Gamma}=\breve{\mathbf{G}}_{j, 1}^{\Gamma} \tilde{\Phi}_{j+1}
$$

form continuous biorthogonal systems

$$
\left\langle\Psi_{j}^{\Gamma}, \tilde{\Psi}_{j}^{\Gamma}\right\rangle=\mathbf{I}, \quad\left\langle\Psi_{j}^{\Gamma}, \tilde{\Phi}_{j}\right\rangle=\left\langle\Phi_{j}, \tilde{\Psi}_{j}^{\Gamma}\right\rangle=\mathbf{0},
$$

with respect to the scalar product (4.1.2). The collections

$$
\Psi^{\Gamma}=\Phi_{0} \bigcup_{j \in \mathbb{N}_{0}} \Psi_{j}^{\Gamma}, \quad \tilde{\Psi}^{\Gamma}:=\tilde{\Phi}_{0} \bigcup_{j \in \mathbb{N}_{0}} \tilde{\Psi}_{j}^{\Gamma}
$$

are biorthogonal bases for $L_{2}(\Gamma)$ :

$$
\left\langle\Psi_{j}^{\Gamma}, \tilde{\Psi}_{j^{\prime}}^{\Gamma}\right\rangle=\delta_{j, j^{\prime}} \mathbf{I}
$$

Proof. The proof is an immediate consequence of the biorthogonality (4.2.6), Proposition 4.3.1, Theorem 4.4.1 and Corollary 3.1 in [5].

Note that the construction (4.4.14) is analogous to the construction of the auxiliary univariate stable completions $\mathbf{M}_{j, 1}$ obtained in Remark 2.4.2, which, in turn, can be used for the initial stable completions $\breve{\mathbf{M}}_{j, 1}^{\Gamma}$ on the manifold used above. 
Efficiency. Obviously the sparseness of the matrices $\breve{\mathbf{M}}_{j}^{\Gamma}, \breve{\mathbf{G}}_{j}^{\Gamma}, \mathbf{M}_{j, 0}^{\Gamma}$ and $\tilde{\mathbf{M}}_{j, \mathbb{0}}^{\Gamma}$ implies the sparseness of the matrices

$$
\mathbf{M}_{j}^{\Gamma}, \mathbf{G}_{j}^{\Gamma}=:\left(\tilde{\mathbf{M}}_{j}^{\Gamma}\right)^{T} .
$$

Thus corresponding multiscale transformations remain efficient. By this we mean that the application of the transformation $\mathbf{T}_{J}$ that takes the array $\mathbf{c}$ of single scale coefficients of any $v_{J}=\mathbf{c}^{T} \Phi_{J}$ into its wavelet coefficients

$$
v_{J}=\mathbf{c}_{j_{0}}^{T} \Phi_{j_{0}}+\sum_{j=j_{0}}^{J-1} \mathbf{d}_{j}^{T} \Psi_{j},
$$

as well as the application of its inverse $\mathbf{T}_{J}^{-1}$, can be carried out at the expense of $\mathcal{O}\left(\# \Phi_{J}\right)$ operations.

Note that (4.4.14) reproduces in the interior of each patch the lifted tensor product biorthogonal wavelets on the cube. For any mesh topology, (4.4.14) produces automatically continuous functions across patch interfaces. In practice, one therefore does not have to assemble the global matrices $\mathbf{M}_{j}^{\Gamma}$. In fact, away from patch boundaries the filters are always the same. One only has to compute locally the entries of $\mathbf{M}_{j, 1}^{\Gamma}$ for $j=j_{0}$ and finitely many cases depending on the topology (or, better, valency of vertices) of the decomposition of $\Gamma$ into the patches $\Gamma_{i}$. Note that the proof of Theorem 4.4.1 indicates how to reduce the evaluation of the matrix products in (4.4.14) to products of tensor product matrices, see (4.4.12). Due to the perfect symmetry properties and the locality of all ingredients it suffices to determine once and for all templates of filter sets referring to different patch topologies around a vertex, as opposed to realizing (4.4.14) for each concrete possibly complex manifold $\Gamma$.

The above construction is illustrated by several figures displaying typical templates of composite wavelets for the piecewise linear or piecewise multilinear case $d=2$. Any higher order examples can be produced in the same fashion. However, the second order accuracy of the trial functions in conjunction with fairly good locality of the basis functions makes this case practically attractive. Note also that in this case one can take $d=\ell=2, \tilde{d}=\tilde{\ell}$ in (2.1.5), (2.1.7), (2.1.8), so that the univariate trial spaces $S\left(\Theta_{j}\right)$ consist of all piecewise linear continuous functions on $[0,1]$ with knots in $\left\{2^{-j} k: k=0, \ldots, 2^{j}\right\}$. Thus, instead of going through the construction of the auxiliary initial stable completions $\breve{\mathbf{M}}_{j, 1}$ from Remark 2.4.2, one can directly identify a suitable $\breve{\mathbf{M}}_{j, 1}$ induced by the hierarchical complement basis which consists of the hat functions corresponding to the new knots on the next higher level.

Figure 4 shows the wavelets produced by (4.4.14), (4.4.15) across the interface bewteen two adjacent intervals. They have cancellation properties of order $\tilde{d}=2$, see Section 4.7 below. Figures 5 and 6 display a stationary interior wavelet as well as wavelets across an interface and near a corner. They have fourth order cancellation properties, $\tilde{d}=4$. As for matrix compression, they are therefore suitable for the single layer potential operator. Recall that asymptotically optimal compression requires the cancellation order to be higher than the order of accuracy of the trial spaces, see (1.1.7). A typical situation that cannot be avoided when dealing, for instance, with closed surfaces is addressed in Figure 7, which shows a wavelet with second order cancellation properties near a singular vertex where only three quadrilateral patches meet. 

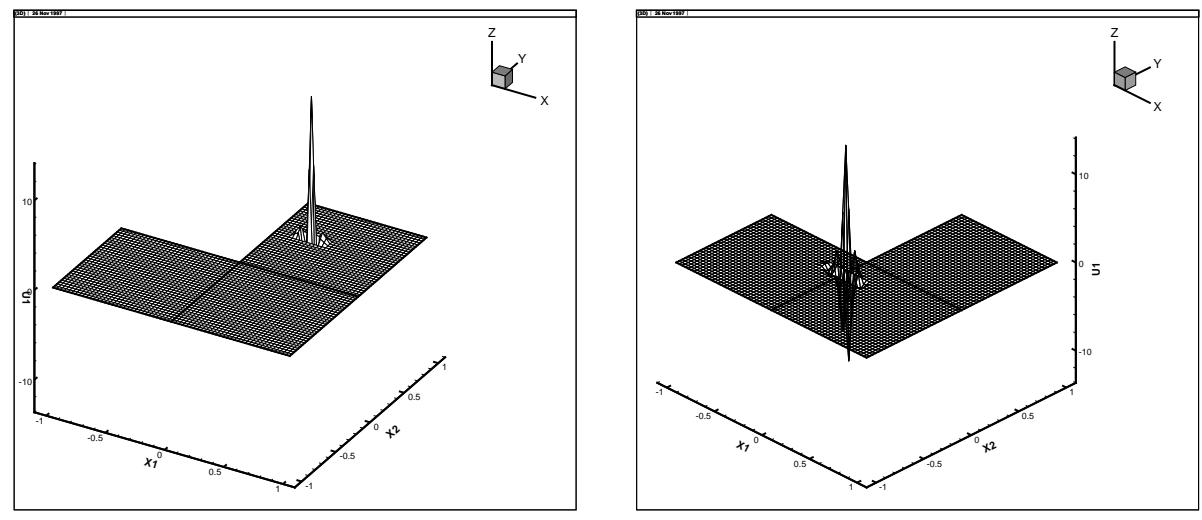

FiguRE 5. Wavelets inside $\Gamma_{i}$ and across $\overline{\Gamma_{1}} \cap \overline{\Gamma_{2}}, d=2, \tilde{d}=4$

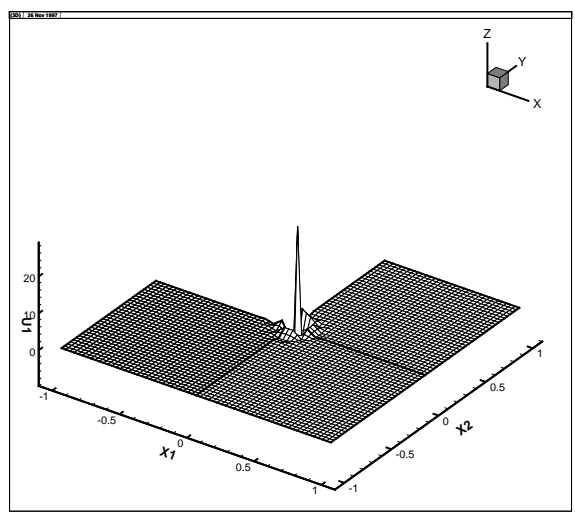

Figure 6 . Wavelets at the corner $\overline{\Gamma_{1}} \cap \overline{\Gamma_{2}} \cap \overline{\Gamma_{3}}, d=2, \tilde{d}=4$

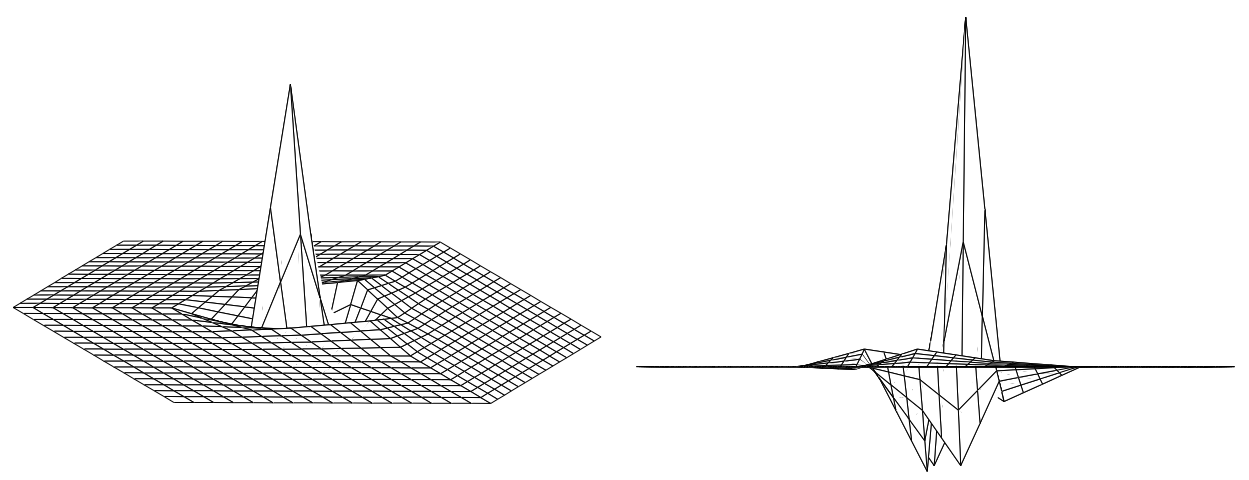

FiguRE 7 . Wavelet near a singular vertex where only 3 edges meet 
4.5. Function spaces on $\Gamma$. We return now to the setting described in Section 4.1 and assume that the manifold $\Gamma$ is of the form (4.1.3) with smooth regular parametrizations $\kappa_{i}, i=1, \ldots, N$. For each $i=1, \ldots, N$, and $m \in \mathbb{N}$, define

$$
\|f\|_{m, i}:=\sum_{|\alpha| \leq m}\left\langle D^{\alpha} f \circ \kappa_{i}, D^{\alpha} f \circ \kappa_{i}\right\rangle_{\square}^{1 / 2} .
$$

Remark 4.5.1. One has for $s \geq 0$

$$
H^{s}(\square)=\left\{f \circ \kappa_{i}: f \in H^{s}\left(\Gamma_{i}\right)\right\}
$$

with

$$
\|f\|_{m, i} \sim\|f\|_{H^{m}\left(\Gamma_{i}\right)}, \quad f \in H^{m}\left(\Gamma_{i}\right), m=0,1,2, \ldots
$$

and

$$
\|f\|_{H^{s}\left(\Gamma_{i}\right)} \sim\left\|f \circ \kappa_{i}\right\|_{H^{s}(\square)}, \quad f \in H^{s}\left(\Gamma_{i}\right), i=1, \ldots, N .
$$

Proof. (4.5.1) follows from the smoothness and regularity of the parametrizations $\kappa_{i}$. The rest of the assertion is confirmed by interpolation arguments.

Moreover, setting $\|\cdot\|_{L_{2}(\Gamma)}^{2}:=\langle\cdot, \cdot\rangle_{\Gamma}$, where $\langle\cdot, \cdot\rangle_{\Gamma}$ denotes the canonical inner product on $\Gamma$, one clearly has that

$$
\|\cdot\|_{0}^{2}:=\langle\cdot, \cdot\rangle \sim\|\cdot\|_{L_{2}(\Gamma)} \cdot
$$

More generally, for $s \geq 0$ define

$$
\|f\|_{s}^{2}:=\sum_{i=1}^{N}\left\|f \circ \kappa_{i}\right\|_{H^{s}(\square)}^{2},
$$

as well as the corresponding dual norms

$$
\|\mid f\|_{-s}:=\sup _{\|g\|_{s}=1}\langle f, g\rangle
$$

and for $s \geq 0$ let $H_{s}$ denote the closure of all globally continuous, piecewise $C^{\infty}$ functions on $\Gamma$ with respect to the norm $\||\cdot|\|_{s}$ while $H_{-s}$ is the dual of $H_{s}$ with respect to the scalar product $\langle\cdot, \cdot\rangle$.

Depending on the global regularity of the manifold $\Gamma$, there may be a limited range for which $H^{s}(\Gamma)$ is well-defined. In the following we will assume that this is the case for $s<s_{\Gamma}$. We will use the following observation.

Remark 4.5.2. $H^{s}(\Gamma)$ is a closed subspace of $H_{s}$ for $0 \leq s \leq s_{\Gamma}$, i.e.,

$$
\|\| v\left\|_{s} \sim\right\| v \|_{H^{s}(\Gamma)}, \quad v \in H^{s}(\Gamma), 0 \leq s<s_{\Gamma} .
$$

Moreover,

$$
H^{s}(\Gamma) \asymp H_{s} \text { for } s \in\left(-1 / 2, \min \left\{3 / 2, s_{\Gamma}\right\}\right) ;
$$

that is, in this range both spaces agree as sets and have equivalent norms.

Proof. It is clear that for $s \geq 0$ we have $\|v\|_{H^{s}(\Gamma)} \lesssim\|v\| \|_{s}, v \in H^{s}(\Gamma)$. Conversely, by extension, $\|v\|_{H^{s}\left(\Gamma_{i}\right)} \leq\|v\|_{H^{s}(\Gamma)}$ for $i=1, \ldots, N$. Moreover, globally continuous piecewise smooth functions belong to $H^{s}(\Gamma)$ for $s<\min \left\{s_{\Gamma}, 3 / 2\right\}$. Thus $H_{s} \subseteq$ $H^{s}(\Gamma)$ in that range, which confirms the upper limit in (4.5.6). The lower limit is caused by the fact that $H_{s}$ and $H^{s}(\Gamma)$ are defined with respect to different inner products. 
4.6. Stability and norm equivalences. Again, the key to the stability properties of the wavelet bases constructed above are appropriate direct and inverse estimates. Due to the stability of the $\Phi_{j}$ and the $\tilde{\Phi}_{j}$, Lemma 2.1 in [14] ensures the following fact.

Remark 4.6.1. The mappings

$$
Q_{j} f:=\left\langle f, \tilde{\Phi}_{j}\right\rangle \Phi_{j}, \quad Q_{j}^{*} f:=\left\langle f, \Phi_{j}\right\rangle \tilde{\Phi}_{j},
$$

are uniformly $L_{2}(\Gamma)$-bounded projectors onto the spaces $S\left(\Phi_{j}\right), S\left(\tilde{\Phi}_{j}\right) \subset C(\Gamma)$, respectively, so that

$$
\begin{aligned}
\left\|f-Q_{j} f\right\|_{L_{2}(\Gamma)} & \lesssim \inf _{v_{j} \in S\left(\Phi_{j}\right)}\left\|f-v_{j}\right\|_{L_{2}(\Gamma)} \\
\left\|f-Q_{j}^{*} f\right\|_{L_{2}(\Gamma)} & \lesssim \inf _{v_{j} \in S\left(\tilde{\Phi}_{j}\right)}\left\|f-v_{j}\right\|_{L_{2}(\Gamma)}
\end{aligned}
$$

To quantify the approximation properties of the spaces $S\left(\Phi_{j}\right), S\left(\tilde{\Phi}_{j}\right)$ we resort to the projectors $P_{j}^{\square}$ from (3.2.1), and define the mapping $P_{j}^{\Gamma}$ by

$$
\left.P_{j}^{\Gamma} f\right|_{\Gamma_{i}}:=\left(P_{j}^{\square}\left(f \circ \kappa_{i}\right)\right) \circ \kappa_{i}^{-1}=\left\langle f \circ \kappa_{i}, \tilde{\Lambda}_{j}^{\square}\right\rangle_{\square} \Theta_{j}^{\square} \circ \kappa_{i}^{-1}, \quad i=1, \ldots, N .
$$

Analogously, we can define $\tilde{P}_{j}^{\Gamma}$ (see Lemma 3.2.2).

Lemma 4.6.1. The $P_{j}^{\Gamma}, \tilde{P}_{j}^{\Gamma}$ are projectors from $C(\Gamma)$ onto $S\left(\Phi_{j}\right), S\left(\tilde{\Phi}_{j}\right)$, respectively. Moreover, one has for $n / 2<d, \tilde{d}$

$$
\left\|f-P_{j}^{\Gamma} f\right\|_{L_{2}(\Gamma)} \lesssim 2^{-j d}\|\| f \|_{d}, \quad f \in H_{d},
$$

and

$$
\left\|f-P_{j}^{\Gamma} f\right\|_{L_{2}(\Gamma)} \lesssim 2^{-j d}\|f\|_{H^{d}(\Gamma)}, \quad f \in H^{d}(\Gamma),
$$

provided that $n / 2<d \leq s_{\Gamma}$. Moreover, completely analogous estimates hold when $P_{j}^{\Gamma}, d$ are replaced by $\tilde{P}_{j}^{\Gamma}, \tilde{d}$.

Proof. Since by Lemma 3.2.1, for $\xi$ as above in (4.2.3), $r=r(\xi)$,

$$
\left\langle f \circ \kappa_{i_{1}}, \tilde{\lambda}_{j, k^{1}}^{\square}\right\rangle_{\square}=\cdots=\left\langle f \circ \kappa_{i_{r}}, \tilde{\lambda}_{j, k^{r}}^{\square}\right\rangle_{\square},
$$

and likewise for $\lambda_{j, k}^{\square}$, we can define the collection of functionals $\tilde{\Lambda}_{j}^{\Gamma}$ by identifying those functionals in the local collections defined by $\left\langle f, \tilde{\Lambda}_{j, i}\right\rangle_{i}:=\left\langle f \circ \kappa_{i}, \tilde{\Lambda}_{j}^{\square}\right\rangle_{\square}$ which correspond to the same points in $\partial \square_{j, i}$. We note then that

$$
P_{j}^{\Gamma} f=\left\langle f, \tilde{\Lambda}_{j}\right\rangle \Phi_{j},
$$

which implies that $P_{j}^{\Gamma}$ maps $C(\Gamma)$ onto $S\left(\Phi_{j}\right)$. Defining $\Lambda_{j}^{\Gamma}$ analogously with a proper normalization matching $(4.2 .5)$, the analogous claim for $\tilde{P}_{j}^{\Gamma}$ follows as well.

Since

$$
\|\| f-P_{j}^{\Gamma} f\left\|_{0, i}=\right\| f \circ \kappa_{i}-P_{j}^{\square}\left(f \circ \kappa_{i}\right) \|_{L_{2}(\square)},
$$

the error bounds follow again by Lemmata 3.2.1 and 3.2.2 and Remarks 4.5.1 and 4.5.2.

Combining Lemma 4.6.1 with Remark 4.6.1 and standard interpolation arguments yields 
Corollary 4.6.1. One has the following error bounds:

$$
\left\|f-Q_{j} f\right\|_{L_{2}(\Gamma)} \lesssim 2^{-j s}\left|\|f \mid\|_{d}, \quad f \in H_{s}, 0 \leq s \leq d .\right.
$$

Moreover, when $n / 2<d, s_{\Gamma}$, one has

$$
\left\|f-Q_{j} f\right\|_{L_{2}(\Gamma)} \lesssim 2^{-j s}\|f\|_{H^{s}(\Gamma)}, \quad f \in H^{s}(\Gamma), 0 \leq s \leq \min \left\{d, s_{\Gamma}\right\} .
$$

Again, completely analogous estimates hold when $Q_{j}, d$ are replaced by $Q_{j}^{*}, \tilde{d}$.

We are now prepared to establish the following norm equivalences.

Theorem 4.6.1. The bases $\Psi^{\Gamma}, \tilde{\Psi}^{\Gamma}$ constructed in Theorem 4.4.2 are Riesz bases for $L_{2}(\Gamma)$. Moreover, setting for simplicity $\Psi_{j_{0}-1}^{\Gamma}:=\Phi_{j_{0}}, \tilde{\Psi}_{j_{0}-1}^{\Gamma}:=\tilde{\Phi}_{j_{0}}$ and $\nabla_{j_{0}-1}^{\Gamma}:=\square_{j_{0}-1}$, one has

$$
\||| f \mid\|_{s} \sim\left(\sum_{j=j_{0}-1}^{\infty} \sum_{\xi \in \nabla_{j}^{\Gamma}} 2^{2 s j}\left|\left\langle f, \tilde{\psi}_{j, \xi}^{\Gamma}\right\rangle\right|^{2}\right)^{1 / 2}, \quad s \in(-\tilde{\gamma}, d-1 / 2),
$$

where $\tilde{\gamma}$ is defined in (3.2.10). In terms of Sobolev norms one has

$$
\|f\|_{H^{s}(\Gamma)} \sim\left(\sum_{j=j_{0}-1}^{\infty} \sum_{\xi \in \nabla_{j}^{\Gamma}} 2^{2 s j}\left|\left\langle f, \tilde{\psi}_{j, \xi}^{\Gamma}\right\rangle\right|^{2}\right)^{1 / 2}, \quad s \in\left(-1 / 2, \min \left\{3 / 2, s_{\Gamma}\right\}\right),
$$

where it is understood that $H^{s}(\Gamma)=\left(H^{-s}(\Gamma)\right)^{*}$ for $s<0$.

Proof. From Lemma 3.2.3 and (4.5.2) one infers that

$$
||\left|v_{j}\right|||_{s} \lesssim 2^{j s}||\left|v_{j}\right| \|_{0}, \quad v_{j} \in V_{j},
$$

where

$$
s< \begin{cases}d-1 / 2=\gamma, & V_{j}=S\left(\Phi_{j}\right), \\ \tilde{\gamma}, & V_{j}=S\left(\tilde{\Phi}_{j}\right) .\end{cases}
$$

On account of Corollary 4.6.1 the claim follows from Remark 4.5.2 combined with the results in [10].

4.7. Cancellation property. By construction, those wavelets in $\Psi^{\Gamma}$ which are supported in a single patch $\Gamma_{i}$ have vanishing moments of order $\tilde{d}$. Here we exploit the fact that the univariate ingredients allow us to choose $\tilde{d}$, in principle, as large as we wish. This is no longer true for those wavelets whose supports intersect several patches, since their restriction to each individual patch is generally no longer a wavelet. However, we will point out next that these wavelets still exhibit cancellation properties which give rise to the same type of estimates needed for the analysis of matrix compression.

To explain the argument that works in all cases, for any projectors $Q, Q^{\prime}$ acting on functions defined on $\Gamma$ denote by $Q \otimes Q^{\prime}$ the projector defined by the action of $Q$ and $Q^{\prime}$ on the first respectively second group of variables of $v: \Gamma \times \Gamma \rightarrow \mathbb{R}$, leaving the other variables fixed. One readily checks now that the Boolean sum $Q \oplus Q^{\prime}:=I \otimes Q+Q^{\prime} \otimes I-Q \otimes Q^{\prime}$ is a projector satisfying

$$
I-Q \oplus Q^{\prime}=(I-Q) \otimes\left(I-Q^{\prime}\right) .
$$


Specifically, for $\lambda, \mu \in \nabla^{\Gamma},|\lambda|=j,|\mu|=l$ and $\left(\psi_{\lambda}^{\Gamma} \otimes \psi_{\mu}^{\Gamma}\right)(\mathbf{x}, \mathbf{y}):=\psi_{\lambda}^{\Gamma}(\mathbf{x}) \psi_{\mu}^{\Gamma}(\mathbf{y})$, one has

$$
\left(Q_{j} \oplus Q_{l}\right)\left(\psi_{\lambda}^{\Gamma} \otimes \psi_{\mu}^{\Gamma}\right)=0
$$

Moreover, since $Q_{j}^{*} \tilde{P}_{j}^{\Gamma}=\tilde{P}_{j}^{\Gamma}$ one has $I-Q_{j}^{*}=\left(I-Q_{j}^{*}\right)\left(I-\tilde{P}_{j}^{\Gamma}\right)$, which, in turn, yields

$$
I-Q_{j}^{*} \oplus Q_{l}^{*}=\left(I-Q_{j}^{*} \oplus Q_{l}^{*}\right)\left(I-\tilde{P}_{j}^{\Gamma} \oplus \tilde{P}_{l}^{\Gamma}\right) .
$$

Now consider the inner product

$$
\langle\langle v, w\rangle\rangle:=\sum_{1 \leq i, i^{\prime} \leq N}\langle\langle v, w\rangle\rangle_{i, i^{\prime}},
$$

where $\langle\langle v, w\rangle\rangle_{i, i^{\prime}}:=\int_{\square} \int_{\square} v\left(\kappa_{i}(x), \kappa_{i^{\prime}}(y)\right) d x d y$. Of course, $Q_{j}^{*} \oplus Q_{l}^{*}$ is the adjoint of $Q_{j} \oplus Q_{l}$ with respect to $\langle\langle\cdot, \cdot\rangle\rangle$. Thus, by (4.7.2) and (4.7.3), one has

$$
\begin{aligned}
\left|\left\langle\left\langle v, \psi_{\lambda}^{\Gamma} \otimes \psi_{\mu}^{\Gamma}\right\rangle\right\rangle\right| & =\left|\left\langle\left\langle\left(I-Q_{j}^{*} \oplus Q_{l}^{*}\right)\left(I-\tilde{P}_{j}^{\Gamma} \oplus \tilde{P}_{l}^{\Gamma}\right) v, \psi_{\lambda}^{\Gamma} \otimes \psi_{\mu}^{\Gamma}\right\rangle\right\rangle\right| \\
& \lesssim\left\|\left(I-Q_{j}^{*} \oplus Q_{l}^{*}\right)\left(I-\tilde{P}_{j}^{\Gamma} \oplus \tilde{P}_{l}^{\Gamma}\right) v\right\|_{L_{2}\left(\Omega_{\lambda} \times \Omega_{\mu}\right)} \\
& \lesssim\left\|\left(I-\tilde{P}_{j}^{\Gamma} \oplus \tilde{P}_{l}^{\Gamma}\right) v\right\|_{L_{2}\left(\hat{\Omega}_{\lambda} \times \hat{\Omega}_{\mu}\right)},
\end{aligned}
$$

where $\Omega_{\lambda}:=\operatorname{supp} \psi_{\lambda}^{\Gamma}$ and, due to the compact supports of the elements in $\Psi, \tilde{\Psi}$ (see (2.3.8)), the $\hat{\Omega}_{\lambda}$ are somewhat larger domains such that $\operatorname{diam} \hat{\Omega}_{\lambda} / \operatorname{diam} \Omega_{\lambda}$ stay uniformly bounded. Now let $\sigma \subseteq \hat{\Omega}_{\lambda} \times \hat{\Omega}_{\mu}$ be any subset which is contained in a single patch product $\Gamma_{i} \times \Gamma_{i^{\prime}}$, say. Since the functionals in $\Lambda_{j}^{\Gamma}$ defining the projectors $\tilde{P}_{j}^{\Gamma}$ (see (4.6.7)) whose supports intersect $\sigma$ are supported either on the patch boundary or inside the patch, the same type of arguments as used in the proof of Lemma 3.2.1 combined with (4.7.1) yield

$$
\left\|\left(I-\tilde{P}_{j}^{\Gamma} \oplus \tilde{P}_{l}^{\Gamma}\right) v\right\|_{L_{2}(\sigma)} \lesssim 2^{-j\left(\tilde{d}+\frac{n}{2}\right)} 2^{-l\left(\tilde{d}+\frac{n}{2}\right)} \max _{|\alpha|,|\beta|=\tilde{d}}\left\|\partial_{x}^{\alpha} \partial_{y}^{\beta} v\right\|_{L_{\infty}(\hat{\sigma})},
$$

where again $\hat{\sigma} \subset \Gamma_{i} \times \Gamma_{i^{\prime}}, \sigma \subseteq \hat{\sigma}$, is a somewhat larger domain whose diameter stays asymptotically proportional to that of $\Omega_{\lambda} \times \Omega_{\mu}$. Thus, when $A$ has the form (1.1.4), note that $\left\langle A \psi_{\mu}^{\Gamma}, \psi_{\lambda}^{\Gamma}\right\rangle=\left\langle\left\langle K, \psi_{\lambda}^{\Gamma} \otimes \psi_{\mu}^{\Gamma}\right\rangle\right\rangle$, so that, when $\Omega_{\lambda} \cap \Omega_{\mu}=\emptyset$, combining (4.7.5) for $K=v$ with the Calderón-Zygmund estimates (1.1.5) yields

$$
\left|\left\langle A \psi_{\mu}^{\Gamma}, \psi_{\lambda}^{\Gamma}\right\rangle\right| \lesssim \frac{2^{-(j+l)(\tilde{d}+n / 2)}}{\operatorname{dist}\left(\Omega_{\lambda}, \Omega_{\mu}\right)^{n+2 \tilde{d}+2 t}},
$$

which is the main foundation of compression estimates commonly derived directly from vanishing moment properties; see $[16,17,23,25]$. Note, however, that here the modified inner product $\langle\cdot, \cdot\rangle$ from $(4.1 .2)$ is used.

4.8. Boundary conditions. When $\Gamma$ is a domain with boundary and $A$ is a second order elliptic operator, one has to specify boundary conditions. We will briefly indicate now how to incorporate in the multiresolution spaces (homogeneous zero order) Dirichlet boundary conditions on some part $\partial \Gamma_{D}$ which is always assumed to be a union of patch boundaries. To clarify ideas, assume that for some $\Gamma_{i}$ the face $\kappa_{i}\left(\{0\} \times[0,1]^{n-1}\right) \subseteq \partial \Gamma_{D}$. Clearly, multiresolution spaces contained in $H_{0,\{0\} \times[0,1]^{n-1}}^{1}(\square)$, the closure in $H^{1}$ of the $C^{\infty}$ functions vanishing on 

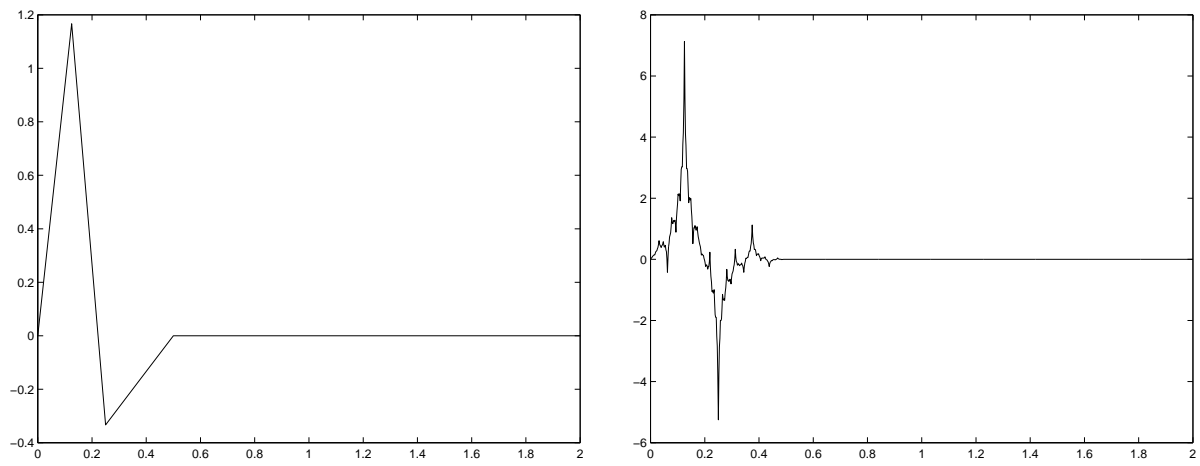

Figure 8. Primal and dual wavelets at the boundary in $H_{0}^{1}([0,1])$

$\{0\} \times[0,1]^{n-1}$, are obtained by replacing the first component $\Theta_{j}$ in the tensor product generator bases by the sets $\Theta_{j}^{0}$ obtained by discarding the function $\theta_{j, \ell-d}$, see $(2.2 .8)$.

Now there are several possibilities to construct suitable biorthogonal generator bases. The first one, suggested by the preceding development, is to discard also the corresponding 'first' function in $\tilde{\Theta}_{j}$. Clearly $\Theta_{j}^{0}$ and $\tilde{\Theta}_{j}^{0}$ are still biorthogonal. By Proposition 2.2.2 and the nature of the refinement relations derived in [14], the resulting spaces are still refinable and satisfy the right boundary conditions on the face of $\square$. The refinement matrices $\mathbf{M}_{j, 0}^{0}, \tilde{\mathbf{M}}_{j, 0}^{0}$ for $\Theta_{j}^{0}, \tilde{\Theta}_{j}^{0}$ are obtained from $\mathbf{M}_{j, 0}, \tilde{\mathbf{M}}_{j, 0}$ by discarding the first row and column.

As for the construction of corresponding wavelets, one should note that the auxiliary stable completions $\breve{\mathbf{M}}_{j, 1}$ constructed in Remark 2.4.2 already correspond to complement basis functions which vanish at the end points of the interval. Thus, one only has to replace the respective factors $\mathbf{M}_{j, 0}$ in $\mathbf{M}_{j, \mathbb{Q}}^{\square}$ (and hence in $\mathbf{M}_{j, \mathbb{Q}}^{\Gamma}$ ) while keeping $\breve{\mathbf{M}}_{j, 1}^{\Gamma}$, and (4.4.14) produces wavelets with appropriate boundary conditions. A simple univariate example again for $d=\tilde{d}=2$ is shown in Figure 8. In agreement with Remark 2.4.1, at least one wavelet does not vanish at the boundary in the unconstrained case displayed in Figure 9. It is now obvious how to implant these ingredients into the general construction in order to realize, for instance, also mixed boundary conditions.

However, again, if one aims at realizing norm equivalences for a range of Sobolev indices $s$ including $-1 / 2$ this approach is not adequate. In fact, the dual of $H_{0, \partial \Gamma_{D}}^{1}$ should have no boundary constraints on $\partial \Gamma_{D}$. Hence the dual multiresolution should have no boundary constraints there, since they should give rise to norm equivalences for $s>1 / 2$ for the unconstrained Sobolev spaces, which is impossible with boundary constrained multiresolution spaces. The problem can also directly be seen by noting that the right hand side data $\left\langle f, \psi_{\lambda}^{\Gamma}\right\rangle$ refer to an expansion with respect to the dual basis $\tilde{\Psi}^{\Gamma}$. When the basis functions in $\tilde{\Psi}^{\Gamma}$ also have zero boundary conditions this representation is at most accurate of order $1 / 2$.

A conceptually better alternative is to replace the reduced sets $\tilde{\Theta}_{j}^{0}$ in the above approach by other biorthogonal bases without zero boundary conditions constructed in [18]. A direct substitution of these ingredients in the above construction would 

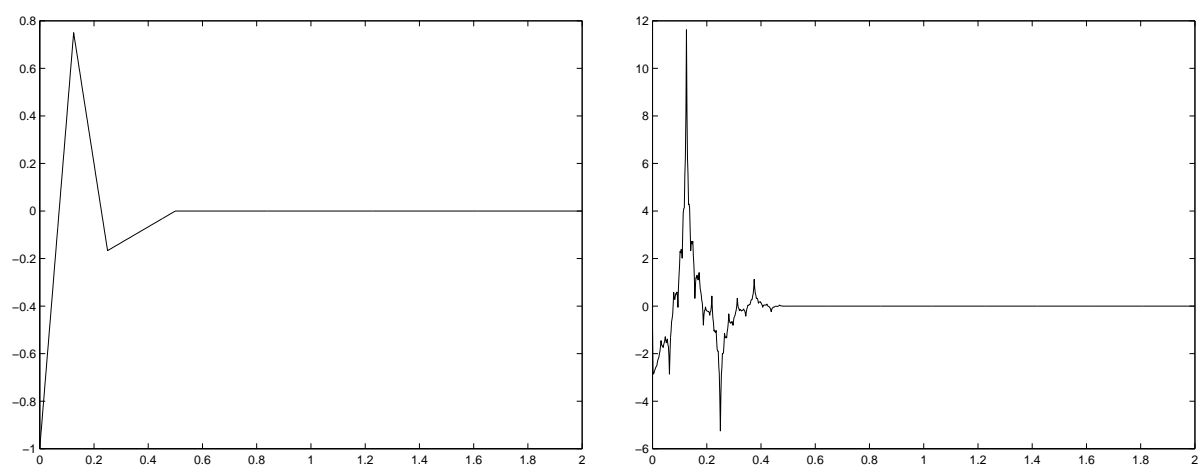

Figure 9. Primal and dual wavelets in $H^{1}([0,1])$ at the boundary

still work and yield bases with the desired boundary conditions on the primal side while avoiding the above mentioned principal deficiencies.

\section{APPENDIX}

Proof of Proposition 2.2.2. By biorthogonality (2.1.2) of the interior functions, (2.2.8) follows from (2.2.4). Since by $(2.2 .3)$

$$
\left\langle\Theta_{X, j}, \tilde{\Theta}_{X, j}\right\rangle_{[0,1]}=\mathbf{C}_{X}\left\langle\Theta_{j}^{X}, \tilde{\Theta}_{j}^{X}\right\rangle_{[0,1]} \tilde{\mathbf{C}}_{X}^{T},
$$

the independence of $\mathbf{C}_{X}, \tilde{\mathbf{C}}_{X}$ of $j$ is a consequence of the above remarks on the matrices $\mathbf{T}_{X}$.

Furthermore, by symmetry (see (2.1.9))

$$
\mathbf{T}_{R}=\mathbf{T}_{L}^{\uparrow}
$$

Thus $\mathbf{C}_{R}=\mathbf{C}_{L}^{\uparrow}, \tilde{\mathbf{C}}_{R}=\tilde{\mathbf{C}}_{L}^{\uparrow}$, which confirms (2.2.2), and it suffices to determine $\mathbf{C}_{L}, \tilde{\mathbf{C}}_{L}$.

To this end, consider the singular value decomposition $\mathbf{T}_{L}=\mathbf{U}^{T} \Sigma \mathbf{V}$, where $\mathbf{U}, \mathbf{V}$ are orthogonal matrices and $\Sigma$ is a diagonal matrix containing the singular values $\sigma_{i}$ of $\mathbf{T}_{L}$ ordered according to size, $\sigma_{i} \geq \sigma_{i+1}$. By (2.2.1), all singular values are strictly greater than zero. One easily checks that for any invertible matrix $\mathbf{R}$ the matrices

$$
\mathbf{C}_{L}:=\mathbf{R} \Sigma^{-1 / 2} \mathbf{U}, \quad \tilde{\mathbf{C}}_{L}:=\mathbf{R}^{-T} \Sigma^{-1 / 2} \mathbf{V},
$$

satisfy

$$
\mathbf{C}_{L} \mathbf{T}_{L} \tilde{\mathbf{C}}_{L}^{T}=\mathbf{I},
$$

which, on account of (A.1), means that the corresponding sets $\Theta_{X, j}, \tilde{\Theta}_{X, j}$ are biorthogonal (see (2.2.4)). Thus it remains to choose $\mathbf{R}$ so that the boundary conditions (2.2.8) hold. Let $\Theta_{j}^{L}(0)$ denote the vector whose entries are the elements of $\Theta_{j}^{L}$ evaluated at zero. By construction one has

$$
\Theta_{j}^{L}(0)=\tilde{\Theta}_{j}^{L}(0)=2^{j / 2} \mathbf{e}^{1},
$$

where $\left(\mathbf{e}^{1}\right)_{i}=\delta_{1, i}$ is the first coordinate vector. By the definition (2.2.3) and (A.5), one has to find a constant $b$ such that

$$
\Theta_{L, j}(0)=\mathbf{C}_{L} \Theta_{j}^{L}(0)=2^{j / 2} \mathbf{R} \Sigma^{-1 / 2} \mathbf{U}^{1}=2^{j / 2} \mathbf{e}^{1}
$$


and

$$
\tilde{\Theta}_{L, j}(0)=\tilde{\mathbf{C}}_{L} \tilde{\Theta}_{j}^{L}(0)=2^{j / 2} \mathbf{R}^{-T} \Sigma^{-1 / 2} \mathbf{V}^{1}=b 2^{j / 2} \mathbf{e}^{1},
$$

where $\mathbf{U}^{1}, \mathbf{V}^{1}$ is the first column of $\mathbf{U}, \mathbf{V}$, respectively. Thus the desired boundary conditions are equivalent to

$$
\mathbf{R} \Sigma^{-1 / 2} \mathbf{U}^{1}=\mathbf{e}^{1}
$$

and

$$
\Sigma^{-1 / 2} \mathbf{V}^{1}=b \mathbf{R}^{T} \mathbf{e}^{1}
$$

Now we make the ansatz

$$
\mathbf{R}:=\left(\begin{array}{c}
a\left(\mathbf{V}^{1}\right)^{T} \Sigma^{-1 / 2} \\
\mathbf{U}_{0}^{T} \Sigma^{1 / 2}
\end{array}\right)
$$

where $a$ is some constant and $\mathbf{U}_{0}$ is the submatrix of $\mathbf{U}$ obtained by discarding the first column. Since $\mathbf{U}$ is an orthogonal matrix, it is clear that

$$
\mathbf{R} \Sigma^{-1 / 2} \mathbf{U}^{1}=a \tau \mathbf{e}^{1} .
$$

Since

$$
\mathbf{T}_{L}^{-1}=\mathbf{V}^{T} \Sigma^{-1} \mathbf{U}
$$

we conclude that

$$
\tau:=\left(\mathbf{T}_{L}^{-1}\right)_{1,1},
$$

and note that, by (A.2), $\tau \neq 0$. From the definition of $\mathbf{R},($ A.7) and (A.8) we infer that $b=\tau^{-1}=\operatorname{det} \mathbf{T}_{L} / \operatorname{det} \mathbf{T}_{L}^{0}$ as claimed. The asserted stability properties (2.2.7) are an immediate consequence of the biorthogonality and the local supports of the elements in $\Theta_{j}, \tilde{\Theta}_{j}$; see Lemma 2.1 in [14].

Proof of Proposition 2.3.1. Let us denote by $\mathbf{I}^{\rightarrow}$ the permutation matrix whose only nonzero entries are on the antidiagonal and have the value 1 . Thus for any matrix $\mathbf{M}$ one has $\mathbf{M}^{\uparrow}=\mathbf{I}^{\rightarrow} \mathbf{M I} \rightarrow$, where $\mathbf{I}^{\rightarrow}$ is always assumed to have the right size without further specification. Since $\left(\mathbf{I}^{\rightarrow}\right)^{2}=\mathbf{I}$, one easily verifies that for any two matrices $\mathbf{A}, \mathbf{B}$ of appropriate sizes

$$
\left(\mathbf{A}^{\downarrow}\right)^{T}=\left(\mathbf{A}^{T}\right)^{\downarrow}, \quad(\mathbf{A B})^{\downarrow}=\mathbf{A}^{\downarrow} \mathbf{B}^{\downarrow}, \quad\left(\mathbf{A}^{-1}\right)^{\uparrow}=\left(\mathbf{A}^{\downarrow}\right)^{-1} .
$$

Now let $\mathbf{N}_{j, 1}, \tilde{\mathbf{N}}_{j, 1}$ denote the submatrix of $\mathbf{M}_{j, 1}^{\prime}, \tilde{\mathbf{M}}_{j, 1}^{\prime}$ consisting of the first $2^{j-1}$ columns, respectively (recall that these matrices always have $2^{j}$ columns). Let

$$
\mathbf{H}_{j, 1}:=\left(\mathbf{N}_{j, 1}, \mathbf{N}_{j, 1}^{\uparrow}\right), \quad \tilde{\mathbf{H}}_{j, 1}:=\left(\tilde{\mathbf{N}}_{j, 1}, \tilde{\mathbf{N}}_{j, 1}^{\uparrow}\right),
$$

and note that by (2.3.7), (2.3.2) and (A.9),

$$
\tilde{\mathbf{M}}_{j, 0}^{T} \mathbf{N}_{j, 1}=\mathbf{0}, \quad\left(\tilde{\mathbf{M}}_{j, 0}^{\uparrow}\right)^{T} \mathbf{N}_{j, 1}^{\uparrow}=\left(\tilde{\mathbf{M}}_{j, 0}^{T} \mathbf{N}_{j, 1}\right)^{\downarrow}=\mathbf{0} .
$$

Applying the same reasoning to $\mathbf{M}_{j, 0}, \tilde{\mathbf{H}}_{j, 1}$ gives us

$$
\tilde{\mathbf{M}}_{j, 0}^{T} \mathbf{H}_{j, 1}=\mathbf{0}, \quad \mathbf{M}_{0,1}^{T} \tilde{\mathbf{H}}_{j, 1}=\mathbf{0} .
$$

The matrices $\mathbf{H}_{j, 1}, \tilde{\mathbf{H}}_{j, 1}$ still have full rank. To see this, consider the collection

$$
\Xi_{j}^{T}:=\Theta_{j+1}^{T} \mathbf{H}_{j, 1}
$$

and note that, by (A.10),

$$
\left\langle\tilde{\Theta}_{j}, \Xi_{j}\right\rangle_{[0,1]}=\tilde{\mathbf{M}}_{j, 0}^{T}\left\langle\tilde{\Theta}_{j+1}, \Theta_{j+1}\right\rangle_{[0,1]}=\tilde{\mathbf{M}}_{j, 0}^{T} \mathbf{H}_{j, 1}=\mathbf{0}
$$


Thus it suffices to show that the elements of $\Xi_{j}$ are linearly independent. Since by construction $\mathbf{H}_{j, 1}=\mathbf{H}_{j, 1}^{\uparrow}$, one has

$$
\left(\mathbf{H}_{j, 1}\right)_{k, 2^{j-1}+r}=\left(\mathbf{H}_{j, 1}\right)_{2^{j+1}-\mu(d)-k, 2^{j-1}+1-r}, \quad r=1, \ldots, 2^{j-1} .
$$

Combining this with the symmetry relations in (2.1.9) and Remark 2.3.1, straightforward calculations yield

$$
\xi_{j, 2^{j-1}+k}(x)=\xi_{j, 2^{j-1}+1-k}(1-x), \quad k=1, \ldots, 2^{j-1} .
$$

Moreover, by definition, $\xi_{j, k}=\psi_{j, k}, k=1, \ldots, 2^{j-1}$. Thus

$$
\Xi_{j}=\left\{\psi_{j, k}, \psi_{j, k}(1-\cdot): k=1, \ldots, 2^{j-1}\right\} .
$$

Now suppose that $\mathbf{c}^{T} \Xi_{j}=0$. Then

$$
\left\langle\mathbf{c}^{T} \Xi_{j}, \tilde{\psi}_{j, k}\right\rangle_{[0,1]}=c_{k}
$$

for $k=1, \ldots, m$, where $m$ is the first column in $\mathbf{M}_{j, 1}$ (and hence in $\mathbf{H}_{j, 1}$ ), which corresponds to the stationary interior masks, so that $\psi_{j, m}$ is fully supported in $[0,1]$. If $j$ is large enough the support of $\tilde{\psi}_{j, m}$ will not overlap any of the supports of the functions $\psi_{j, k}(1-\cdot), k=1, \ldots, 2^{j-1}$, so that (A.12) indeed follows from biorthogonality and gives $c_{k}=0$, for $k=1, \ldots, m$. Likewise, testing with the functions $\tilde{\psi}_{j, k}(1-\cdot)$ for $k=1, \ldots, m$ ensures that also $c_{k}=0$ for $k=2^{j}, \ldots, 2^{j}-$ $m+1$. Therefore

$$
\mathbf{c}^{T} \Xi_{j}=\sum_{k=m+1}^{2^{j}-m} c_{k} \xi_{j, k}=0
$$

is a linear combination of functions which are supported in $(0,1)$. Now note from the construction in [14] that for $d$ odd the first column of $\mathbf{N}_{j, 1}^{\uparrow}$ has the same support as the $2^{j-1}+1$ st column of $\mathbf{M}_{j, 1}^{\prime}$. Thus the functions in the above linear combination all have pairwise different supports. Therefore (A.13) implies that $\mathbf{c}=\mathbf{0}$, which confirms our claim.

Now consider

$$
\begin{aligned}
\tilde{\mathbf{H}}_{j, 1}^{T} \mathbf{H}_{j, 1} & =\left(\begin{array}{c}
\tilde{\mathbf{N}}_{j, 1}^{T} \\
\left(\tilde{\mathbf{N}}_{j, 1}^{\uparrow}\right)^{T}
\end{array}\right)\left(\mathbf{N}_{j, 1}, \mathbf{N}_{j, 1}^{\uparrow}\right) \\
& =\left(\begin{array}{cc}
\mathbf{I} & \tilde{\mathbf{N}}_{j, 1}^{T} \mathbf{N}_{j, 1}^{\uparrow} \\
\left(\tilde{\mathbf{N}}_{j, 1}^{\uparrow}\right)^{T} \mathbf{N}_{j, 1} & \mathbf{I}
\end{array}\right)=: \mathbf{K}_{j} .
\end{aligned}
$$

Since by (A.9)

$$
\tilde{\mathbf{N}}_{j, 1}^{T} \mathbf{N}_{j, 1}^{\uparrow}=\left(\left(\tilde{\mathbf{N}}_{j, 1}^{\uparrow}\right)^{T} \mathbf{N}_{j, 1}\right)^{\downarrow}
$$

the matrix $\mathbf{K}_{j}$ has the form

$$
\mathbf{K}_{j}=\left(\begin{array}{cc}
\mathbf{I} & \mathbf{A}_{j} \\
\mathbf{A}_{j}^{\uparrow} & \mathbf{I}
\end{array}\right)
$$

where

$$
\mathbf{A}_{j}=\left(\begin{array}{cc}
\mathbf{0} & \mathbf{0} \\
\mathbf{a} & \mathbf{0}
\end{array}\right), \quad \mathbf{A}_{j}^{\uparrow}=\left(\begin{array}{cc}
\mathbf{0} & \mathbf{a} \downarrow \\
\mathbf{0} & \mathbf{0}
\end{array}\right)
$$


and the block $\mathbf{a}$ is independent of $j$. By the above remarks $\mathbf{K}_{j}$ must be invertible. Noting that

$$
\mathbf{A}_{j}^{\uparrow} \mathbf{A}_{j}=\left(\begin{array}{cc}
\mathbf{a} \uparrow \mathbf{a} & \mathbf{0} \\
\mathbf{0} & \mathbf{0}
\end{array}\right), \quad\left(\mathbf{I}-\mathbf{A}_{j}^{\uparrow} \mathbf{A}_{j}\right)^{-1}=\left(\begin{array}{cc}
\left(\mathbf{I}-\mathbf{a} \mathbf{a}^{\uparrow} \mathbf{a}\right)^{-1} & \mathbf{0} \\
\mathbf{0} & \mathbf{I}
\end{array}\right),
$$

one easily confirms that

$$
\mathbf{K}_{j}^{-1}=\left(\begin{array}{cccc}
\mathbf{I} & \mathbf{0} & \mathbf{0} & \mathbf{0} \\
\mathbf{0} & \mathbf{I}+\mathbf{a}\left(\mathbf{I}-\mathbf{a}^{\uparrow} \mathbf{a}\right)^{-1} \mathbf{a}^{\uparrow} & -\mathbf{a}\left(\mathbf{I}-\mathbf{a}^{\uparrow} \mathbf{a}\right)^{-1} & \mathbf{0} \\
\mathbf{0} & -\left(\mathbf{I}-\mathbf{a}^{\uparrow} \mathbf{a}\right)^{-1} \mathbf{a}^{\uparrow} & \left(\mathbf{I}-\mathbf{a}^{\uparrow} \mathbf{a}\right)^{-1} & \mathbf{0} \\
\mathbf{0} & \mathbf{0} & \mathbf{0} & \mathbf{I}
\end{array}\right)
$$

Thus both $\mathbf{K}_{j}$ and $\mathbf{K}_{j}^{-1}$ are banded with band width independent of $j$. Moreover, one easily checks from the definition of $\mathbf{K}_{j}$ and (A.9) that

$$
\mathbf{K}_{j}^{\uparrow}=\mathbf{K}_{j}, \quad\left(\mathbf{K}_{j}^{-1}\right)^{\uparrow}=\mathbf{K}_{j}^{-1} .
$$

Thus, defining

$$
\mathbf{M}_{j, 1}:=\mathbf{H}_{j, 1} \mathbf{K}_{j}^{-1}, \quad \tilde{\mathbf{M}}_{j, 1}:=\tilde{\mathbf{H}}_{j, 1},
$$

we readily infer from (A.10) and (A.14) that (2.3.7) holds. Moreover, since by construction $\tilde{\mathbf{H}}_{j, 1}^{\uparrow}=\tilde{\mathbf{H}}_{j, 1}$ and $\mathbf{H}_{j, 1}^{\uparrow}=\mathbf{H}_{j, 1}$, the assertion follows from (A.14) and (A.9).

\section{ACKNOWLEDGMENTS}

We are indepted to T. Barsch, H. Harbrecht and J. Vorloeper for their assistance in implementing the scheme and preparing the illustrations.

\section{REFERENCES}

1. R.A. Adams, Sobolev Spaces, Academic Press, 1975. MR 56:9247

2. G. Beylkin, R. Coifman and V. Rokhlin, The fast wavelet transform and numerical algorithms, Comm. Pure Appl. Math. 44, 1991, 141-183. MR 92c:65061

3. C. de Boor, A Practical Guide to Splines, Springer, 1978. MR 80a:65027

4. C. Canuto, M.Y. Hussaini, A. Quarteroni, T.A. Zang, Spectral Methods in Fluid Dynamics, Springer Series in Computational Physics, Springer, 1988. MR 89m:76004

5. J.M. Carnicer, W. Dahmen and J.M. Peña, Local decomposition of refinable spaces, Appl. Comp. Harm. Anal. 3, 1996, 127-153. MR 97f:42050

6. A. Cohen, W. Dahmen and R.A. DeVore, Multiscale decompositions on bounded domains, preprint, 1995, to appear in Trans. Amer. Math. Soc.

7. A. Cohen, I. Daubechies and J.-C. Feauveau, Biorthogonal bases of compactly supported wavelets, Comm. Pure Appl. Math. 45, 1992, 485-560. MR 93e:42044

8. A. Cohen, I. Daubechies and P. Vial, Wavelets on the interval and fast wavelet transforms, Appl. Comp. Harm. Anal. 1, 1993, 54-81. MR 94m:42074

9. S. Dahlke, W. Dahmen, R. Hochmuth, R. Schneider, Stable multiscale bases and local error estimation for elliptic problems, Applied Numerical Mathematics 23, 1997, 21-47. MR 98a:65075

10. W. Dahmen, Stability of multiscale transformations, J. Fourier Anal. Appl. 2, 1996, 341361. MR 97i: 46133

11. W. Dahmen, Multiscale analysis, approximation, and interpolation spaces, in: Approximation Theory VIII, C.K. Chui, L.L. Schumaker (eds.), World Scientific Publishing Co., 1995, 47-88. MR 98f:41032

12. W. Dahmen, Wavelet and multiscale methods for operator equations, Cambridge University Press, Acta Numerica 6, 1997, 55-228. MR 98m:65102

13. W. Dahmen, A. Kunoth, Multilevel preconditioning, Numer. Math. 63, 1992, 315-344. MR 93j:65065 
14. W. Dahmen, A. Kunoth and K. Urban, Biorthogonal spline-wavelets on the interval Stability and moment conditions, IGPM Report \# 129, RWTH Aachen, 1996, to appear in Appl. Computational Harm. Anal.

15. W. Dahmen, S. Prößdorf, R. Schneider, Wavelet approximation methods for periodic pseudodifferential equations. Part 1 - Convergence analysis, Math. Zeitschrift 215, 1994, 583-620. MR 95g:65148

16. W. Dahmen, S. Prößdorf, R. Schneider, Wavelet approximation methods for periodic pseudodifferential equations. Part 2 - Fast solution and matrix compression, Advances in Computational Mathematics 1, 1993, 259-335. MR 95g:65149

17. W. Dahmen, S. Prößdorf, R. Schneider, Multiscale methods for pseudo-differential equations on smooth manifolds, in: Proceedings of the International Conference on Wavelets: Theory, Algorithms, and Applications, C.K. Chui, L. Montefusco, L. Puccio (eds.), Academic Press, 1994, 385-424. MR 96c:65208

18. W. Dahmen, R. Schneider, Wavelet bases with complementary boundary conditions Function spaces on the cube, Results in Mathematics, 34, 1998, 255-293.

19. R. Dautray, J.-L. Lions, Mathematical Analysis and Numerical Methods for Science and Technology, Vol. 4, Integral Equations and Numerical Methods, Springer, 1990. MR 91h:00004b

20. K. Höllig and H. Mögerle, G-splines, Comp. Aided Geom. Design 7, 1990, 197-207. MR 91e:65006

21. S. Jaffard, Wavelet methods for fast solution of elliptic problems, SIAM J. Numer. Anal. 29, 1992, 965-987. MR 93j:35042

22. A. Jouini and P.G. Lemarié-Rieusset, Analyses multirésolutions biorthogonales et applications, Ann. Inst. H. Poincaré Anal. Non Linéaire, 10, 1993, 453-471.

23. T. von Petersdorff and C. Schwab, Wavelet approximations of the first kind integral equation on plygons, Numer. Mathematik 74, 1996, 479-516. MR 97m:65223

24. U. Reif, Biquadratic G-spline surfaces, CAGD 12, 1995, 193-205. MR 95k:65022

25. R. Schneider, Multiskalen- und Wavelet-Matrixkompression: Analysisbasierte Methoden zur Lösung großer vollbesetzter Gleichungssysteme, Habilitationsschrift, TH Darmstadt, Germany, 1995 (in German).

26. W. Sweldens, The lifting scheme: A custom-design construction of biorthogonal wavelets, Appl. Comput. Harmon. Anal. 3, 1996, 186-200. MR 97b:42060

Institut für Geometrie und Praktische Mathematik, RWTH Aachen, Templergraben 55, 52056 Aachen, Germany

E-mail address: dahmen@igpm.rwth-aachen.de

URL: http://www.igpm.rwth-aachen.de/ dahmen/

Fakultät Für Mathematik, Technische Universität Chemnitz-Zwickau, 09107 ChemNitz, GeRmany

E-mail address: reinhold.schneider@mathematik.tu-chemnitz.de

URL: http://www.tu-chemnitz.de/ reinhold/ 